\title{
Scale-invariant two-component dark matter
}

\author{
Seyed Yaser Ayazi ${ }^{1, \mathrm{a}}$, Ahmad Mohamadnejad ${ }^{2}$ \\ ${ }^{1}$ Physics Department, Semnan University, P.O. Box. 35131-19111, Semnan, Iran \\ 2 Young Researchers and Elite Club, Islamshahr Branch, Islamic Azad University, Islamshahr 3314767653, Iran
}

Received: 3 November 2018 / Accepted: 4 February 2019 / Published online: 15 February 2019

(c) The Author(s) 2019

\begin{abstract}
We study a scale-invariant extension of the standard model which can simultaneously explain dark matter and the hierarchy problem. In our set-up, we introduce a scalar and a spinor as two-component dark matter in addition to a scalon field as a mediator. An interesting point about our model is that due to scale-invariant conditions, compared to other two-component dark matter models, it has fewer independent parameters. Possible astrophysical and laboratory signatures of a two-component dark matter candidate are explored and it is shown that the highest contribution of observed relic density of dark matter can be determined by spinor dark matter. The detectability of these dark matter particles is studied and direct and invisible Higgs decay experiments are used to rule out part of the parameter space of the model. In addition, the dark matter self-interactions are considered and it is shown that their contributions saturate this constraint in the resonant regions.
\end{abstract}

\section{Introduction}

The standard model (SM) has been established by the discovery of the Higgs boson and it can explain almost all of experimental results obtained until now. However, there are a number of unanswered issues, either theoretical or experimental, such as the hierarchy problem, active neutrino masses, the dark matter (DM) relic abundance, the baryon asymmetry of the Universe, inflation in the early Universe, and dark energy.

The existence of DM is inferred from crucial evidence such as galactic rotation curves, gravitational lensing, observations of merging galaxies, cosmic microwave background (CMB) measurements, the large scale structure of the Universe and collisions of bullet clusters. As mentioned, there is still lack of experimental or observational evidence to precisely distinguish the correct particle physics model for DM physics.

a e-mail: syaser.ayazi@semnan.ac.ir
To explain these issues a number of the SM extensions, such as supersymmetric standard model, technicolor and extra dimension theories, have been proposed. Despite the broad searches as regards beyond SM physics at LHC, null results for beyond SM theories [1-6] show that we have enough motivation to think about alternative theories.

In almost all extended models, there are some additional particles, which usually have heavier masses than the electroweak (EW) scale. It is well known that the hierarchy problem arises from the fact that the negative Higgs mass term in the Lagrangian of the SM causes a quadratical divergent term proportional to the energy scale cut-off $\Lambda^{2}$ after including the quantum corrections. As an idea avoiding the hierarchy problem, classically scale-invariant extensions provide an attractive framework [7-9]. In this picture, it is supposed that the tree-level Higgs mass is zero and at the quantum level the Higgs scalar gains a small mass from the radiative corrections. In fact, the Higgs mass term is the only term that breaks the classical scale invariance in the SM. Note that classical scale invariance by itself does not explain the hierarchy problem; however, it can be regarded as a procedure for model building, which limits the space of Lagrangians to contain only operators with dimensionless coupling constants. The hierarchy problem then reveals itself as the absence of couplings between the Higgs and other energy scales that are dynamically generated in the UV cut-off scale $[10,11]$. Therefore, by regarding this condition, one can practically remove the hierarchy problem.

In recent years, a lot of classically scale-invariant models have been studied for the solution of the hierarchy problem and the DM problem [12-20]. On the other hand, in order to resolve the small-scale problems (through self-interaction) and at the same time explain the potential indirect signals, one needs very different DM masses. Therefore, to interpret both observations, a multi-component DM seems to be a natural possibility. Furthermore, multi-component DM models, besides the standard annihilations and coannihilations, allow one to have conversion, semi-annihilation, and decay 
processes which make the dark sector (thermal) dynamics much more interesting. Therefore, the dynamics of multicomponent DM is much richer than simple WIMP, and it arouses curiosity by itself. In this paper, we study scalarspinor two-component DM, in order to have one candidate for each bosonic and fermionic particles. The possibility of other two-component models without scale invariance has been extensively considered in the literature [21-34]. Also the two-component DM has been studied in the context of scalar WIMP-like candidates [35]. Our goal in this paper is to address the DM relic density and the hierarchy problem by an extension of the scale-invariant standard model (SISM) which contains a scalar and a spinor DM candidate.

The structure of this paper is as follows: in Sect. 2, we introduce the scale-invariant SM with two-component scalar and fermionic DM scenarios. In Sect. 3, we study perturbativity constraints on two-component scale-invariant DM. In Sect. 4, we study freeze-out solutions to the relic density constraint. In Sect. 5, we will study phenomenological aspects such as direct detection, indirect detection, self-interaction and invisible Higgs decay searches on parameter space of our model. The results are summarized in Sect. 6. The decay rate and cross section formulas for the self-interaction of the two components of DM are summarized in the appendix.

\section{The model}

In the SISM, before electroweak symmetry breaking all fields in the scale-invariant sector of potential are massless. At the quantum level these fields gain mass from radiative Coleman-Weinberg symmetry breaking [9].

In this paper, we consider a scale-invariant extension of the SM where the Higgs mass term is absent, and the only term remaining in the Higgs potential will be $\lambda_{H}\left(H^{\dagger} H\right)^{2}$. In order to have a scale-invariant version of the SM possessing a Higgs doublet and other SM particles with their physical masses, at least two more scalars (singlet) must be added to the theory. This arises from the fact that in the absence of scalar DM, the square scalon mass is completely fixed and would be negative [8]. In order to satisfy this condition, we add three new fields, two scalars and one spinor, in our model. All fields are singlets under an SM gauge transformation and they are massless before spontaneous symmetry breaking. Two of these new fields, the scalar $S$ and the spinor $\chi$, are assumed to be odd under a $Z_{2}$ symmetry. This discrete symmetry guarantees the stability of the lightest odd particles. The other scalar field, $\phi$, and all SM particles are even under $Z_{2}$. Therefore under $Z_{2}$ symmetry new fields transform as follows:

$\phi \rightarrow \phi, S \rightarrow-S, \chi \rightarrow-\chi$.
The scalar part of the Lagrangian including the new fields is

$$
\mathcal{L}_{\text {scalar }}=\frac{1}{2} \partial_{\mu} \phi \partial^{\mu} \phi+\frac{1}{2} \partial_{\mu} S \partial^{\mu} S+D_{\mu} H^{\dagger} D^{\mu} H-V(H, \phi, S),
$$

where the most general scale-invariant potential $V(H, \phi, S)$ which is renormalizable and invariant under gauge and $Z_{2}$ symmetry is

$$
\begin{aligned}
V(H, \phi, S)= & \frac{1}{6} \lambda_{H}\left(H^{\dagger} H\right)^{2}+\frac{1}{4 !} \lambda_{\phi} \phi^{4}+\frac{1}{4 !} \lambda_{s} S^{4} \\
& +\lambda_{\phi H} \phi^{2} H^{\dagger} H+\lambda_{s} S^{2} H^{\dagger} H+\lambda_{\phi s} \phi^{2} S^{2}
\end{aligned}
$$

where $H, \phi$ and $S$ are the doublet Higgs, the scalon and DM scalars, respectively.

The scale-invariant terms including the new spinor field and its allowed interaction are given by

$\mathcal{L}_{\text {spinor }}=\bar{\chi}\left(i \gamma^{\mu} \partial_{\mu}-g \phi\right) \chi$.

Since there are no allowed interaction terms in the Lagrangian including both odd fields, the heavier odd particle also turns out to be stable. Therefore, the model has an accidental symmetry that stabilizes the heavier odd particles and it contains two DM candidates.

In unitary gauge, $H=\frac{1}{\sqrt{2}}\left(\begin{array}{l}0 \\ h\end{array}\right)$, the potential (3) becomes

$$
\begin{aligned}
V(h, \phi, S)= & \frac{1}{4 !} \lambda_{H} h^{4}+\frac{1}{4 !} \lambda_{\phi} \phi^{4}+\frac{1}{4 !} \lambda_{s} S^{4} \\
& +\frac{1}{2} \lambda_{\phi H} \phi^{2} h^{2}+\frac{1}{2} \lambda_{s} S^{2} h^{2}+\lambda_{\phi S} \phi^{2} S^{2} .
\end{aligned}
$$

The minimum of potential (5) corresponds to the field vacuum expectation values. The necessary conditions for a local minimum of $V(h, \phi, S)$ are

$$
\begin{aligned}
& \frac{\partial V}{\partial h}=0 \Rightarrow \frac{1}{3 !} \lambda_{H} h^{3}+\lambda_{\phi H} \phi^{2} h+\lambda_{s H} S^{2} h=0, \\
& \frac{\partial V}{\partial \phi}=0 \Rightarrow \frac{1}{3 !} \lambda_{\phi} \phi^{3}+\lambda_{\phi H} \phi h^{2}+2 \lambda_{\phi S} S^{2} \phi=0, \\
& \frac{\partial V}{\partial S}=0 \Rightarrow \frac{1}{3 !} \lambda_{S} S^{3}+\lambda_{S H} S h^{2}+2 \lambda_{\phi s} \phi^{2} S=0 .
\end{aligned}
$$

Equation (6) should hold for the field vacuum expectation values. Note that we require the non-vanishing vacuum expectation values for the fields $h$ and $\phi$ so the scalar field $S$ remains stable because of the $Z_{2}$ symmetry and thereby it can play the role of the DM. Therefore, we put $S=0$ in Eq. (6): 


$$
\begin{aligned}
\frac{1}{3 !} \lambda_{H} h^{3}+\lambda_{\phi H} \phi^{2} h & =0 \\
\frac{1}{3 !} \lambda_{\phi} \phi^{3}+\lambda_{\phi H} \phi h^{2} & =0 .
\end{aligned}
$$

We are looking for a non-trivial solution of (7) corresponding to the non-vanishing vacuum expectation values for $h$ and $\phi$. For non-zero $h$ and $\phi$, Eq. (7) leads to

$$
\left(\begin{array}{cc}
\frac{1}{3 !} \lambda_{H} & \lambda_{\phi H} \\
\lambda_{\phi H} & \frac{1}{3 !} \lambda_{\phi}
\end{array}\right)\left(\begin{array}{l}
h^{2} \\
\phi^{2}
\end{array}\right)=0 \Rightarrow\left|\begin{array}{cc}
\frac{1}{3 !} \lambda_{H} & \lambda_{\phi H} \\
\lambda_{\phi H} & \frac{1}{3 !} \lambda_{\phi}
\end{array}\right|=0
$$

or simply

$\lambda_{H} \lambda_{\phi}=\left(3 ! \lambda_{\phi H}\right)^{2}$.

Note that according to condition (8), the minimum of the potential term $V(h, \phi, S)$ corresponding to the vacuum expectation values of the fields is zero.

The field $H$ breaks the electroweak symmetry with vacuum expectation value $\langle H\rangle=\frac{1}{\sqrt{2}}\left(\begin{array}{c}0 \\ v_{1}\end{array}\right)$, where $v_{1}=$ $246 \mathrm{GeV}$. Thus the Higgs field after spontaneous symmetry breaking is given by

$H=\frac{1}{\sqrt{2}}\left(\begin{array}{c}0 \\ v_{1}+h_{1}\end{array}\right)$.

As mentioned, the field $\phi$ also acquires a vacuum expectation value,

$\phi=v_{2}+h_{2}$.

Notice that $h_{1}$ and $h_{2}$ mix with each other and can be rewritten by the mass eigenstates $H_{1}$ and $H_{2}$ as

$$
\left(\begin{array}{l}
H_{1} \\
H_{2}
\end{array}\right)=\left(\begin{array}{cc}
\cos \alpha & -\sin \alpha \\
\sin \alpha & \cos \alpha
\end{array}\right)\left(\begin{array}{l}
h_{1} \\
h_{2}
\end{array}\right)
$$

where $\alpha$ is the mixing angle. We identify $H_{1}$ with the SM-like Higgs observed at the LHC with a mass of about $125 \mathrm{GeV}$.

After the symmetry breaking, we have the following constraints:

$$
\begin{aligned}
\nu_{2} & =\frac{M_{\chi}}{g}, \\
\sin \alpha & =\frac{\frac{\nu_{1}}{\nu_{2}}}{\sqrt{1+\left(\frac{\nu_{1}}{\nu_{2}}\right)^{2}}}, \\
M_{H_{2}} & =0, \\
\lambda_{H} & =\frac{3 M_{H_{1}}^{2}}{v_{1}^{2}} \cos ^{2} \alpha,
\end{aligned}
$$

$$
\begin{aligned}
\lambda_{\phi} & =\frac{3 M_{H_{1}}^{2}}{v_{2}^{2}} \sin ^{2} \alpha, \\
\lambda_{\phi H} & =-\frac{M_{H_{1}}^{2}}{2 v_{1} \nu_{2}} \sin \alpha \cos \alpha, \\
\lambda_{s H} & =\frac{M_{s}^{2}-2 \lambda_{\phi s} v_{2}^{2}}{v_{1}^{2}},
\end{aligned}
$$

where $M_{S}$ and $M_{\chi}$ are the masses of scalar and spinor DM after symmetry breaking, respectively. The $\mathrm{H}_{2}$ field (scalon) is massless at tree level, and it can be shown that by considering this issue the elastic scattering cross section of DM off nuclei becomes drastically large and the model is immediately excluded by direct detection experiments. However, at one-loop level the scalon gains mass and the direct detection cross section should be computed with the one-loop mass. The one-loop correction gives a mass to the massless eigenstate $H_{2}[8,35]$ :

$M_{H_{2}}^{2}=-\frac{\lambda_{\phi H}}{16 \pi^{2} M_{H_{1}}^{2}}\left(M_{H_{1}}^{4}+M_{s}^{4}+6 M_{W}^{4}+3 M_{Z}^{4}-4 M_{\chi}^{4}-12 M_{t}^{4}\right)$.

Notice that in the absence of scalar and fermionic DM, the scalon mass was completely fixed by the Higgs particle, the $Z$ gauge boson and the top quark masses. For this reason, adding the scalar field is inevitable. Moreover, in the absence of additional scalar DM, the square scalon mass could be negative. Since $M_{H_{2}}^{2}>0$ and $\lambda_{\phi H}<0$, Eq. (14) leads to the following constraint on $M_{s}$ :

$M_{s}>f\left(M_{\chi}\right)$

where

$f\left(M_{\chi}\right)=\sqrt[4]{4 M_{\chi}^{4}-\left(M_{H_{1}}^{4}+6 M_{W}^{4}+3 M_{Z}^{4}-12 M_{t}^{4}\right)}$,

and $f(0)=310.7 \mathrm{GeV}$, which is the minimum of $M_{s}$. Throughout this paper, we satisfy this condition.

According to (13), the model introduces only five free parameters, $\lambda_{s}, \lambda_{\phi s}, M_{s}, M_{\chi}, g$. In addition, the quartic coupling $\lambda_{s}$ is irrelevant to the DM relic density. Therefore, the remaining free parameters are

$\lambda_{\phi s}, M_{s}, M_{\chi}, g$.

It is remarkable that our model, in comparison with other two-component DM models, has a much lower number of independent parameters and behaves like a single-component model. For this reason, it would be difficult to satisfy all theoretical and phenomenological constraints simultaneously. 
This is the point that we encounter in the next sections. In the following, we examine perturbativity constraints on these four parameters.

\section{Theoretical constraints}

In this section, we discuss various constraints on the parameters of our model from theoretical considerations. These are furnished in the following. Perturbativity constraints on the parameters of the Lagrangian are

$$
\begin{aligned}
& -4 \pi<\lambda_{H}, \lambda_{\phi}, \lambda_{s}, \lambda_{\phi H}, \lambda_{s H}, g<4 \pi \\
& -8 \pi<\lambda_{\phi s}<8 \pi .
\end{aligned}
$$

Considering the constraints (13) we have

$0<\frac{3 M_{H_{1}}^{2}}{v_{1}^{2}} \cos ^{2} \alpha<4 \pi$,

$0<\frac{3 M_{H_{1}}^{2}}{v_{2}^{2}} \sin ^{2} \alpha<4 \pi$

$0<\frac{M_{H_{1}}^{2}}{2 v_{1} \nu_{2}} \sin \alpha \cos \alpha<4 \pi$,

$-4 \pi<\frac{M_{s}^{2}-2 \lambda_{\phi s} v_{2}^{2}}{v_{1}^{2}}<4 \pi$,

$0<g<4 \pi$

One can easily show that Eq. (20) and Eq. (22) are established automatically. The constraint (21) leads to

$0<\sin \alpha<\max (\sin \alpha)$

where $\max (\sin \alpha)=\sqrt{\sqrt{A^{2}+2 A}-A}$ with $A=\frac{2 \pi v_{1}^{2}}{3 M_{H_{1}}^{2}}$ $(\max (\sin \alpha)=0.972)$. The above equation, $0<\sin \alpha<$ 0.972 , is not a strong constraint on $\sin \alpha$. However, it leads to a constraint on $M_{\chi}$ :

$M_{\chi}>\frac{\sqrt{1-[\max (\sin \alpha)]^{2}}}{\max (\sin \alpha)} g, v_{1}=(59.38 \mathrm{GeV}) g$

Regarding Eq. (23)

$2 \lambda_{\phi s} v_{2}^{2}-4 \pi v_{1}^{2}<M_{s}^{2}<2 \lambda_{\phi s} v_{2}^{2}+4 \pi v_{1}^{2}$,

and according to (15) we have

$$
\begin{aligned}
f^{2}\left(M_{\chi}\right) & <M_{s}^{2}<2 \lambda_{\phi s} v_{2}^{2}+4 \pi v_{1}^{2} \Rightarrow\left(\frac{f^{2}\left(M_{\chi}\right)}{2}\right)^{2} \\
& <\left(\lambda_{\phi s} v_{2}^{2}+2 \pi v_{1}^{2}\right)^{2} .
\end{aligned}
$$

Considering $f^{4}\left(M_{\chi}\right)=4 M_{\chi}^{4}+f^{4}(0)$ and $v_{2}=\frac{M_{\chi}}{g}$, Eq. (28) leads to

$a M_{\chi}^{4}-b M_{\chi}^{2}-c<0$,

where $a=1-\frac{\lambda_{\phi s}^{2}}{g^{4}}, b=\frac{4 \pi \lambda_{\phi s} v_{1}^{2}}{g^{2}}$, and $c=4 \pi^{2} v_{1}^{4}-\frac{f^{4}(0)}{4}>0$. For $\lambda_{\phi s}>0(b>0)$, there are two possibilities: first $a<0$, so Eq. (29) is trivial, and second $a>0$, providing a constraint on $M_{\chi}$ :

$M_{\chi}<\sqrt{\frac{b+\sqrt{b^{2}+4 a c}}{2 a}}$.

Finally, we choose the following domains for the parameter space (17):

$0<g<4 \pi$,

$0<\lambda_{\phi s}<8 \pi$,

$(59.38 \mathrm{GeV}) \mathrm{g}<\mathrm{M}_{\chi}<\sqrt{\frac{\mathrm{b}+\sqrt{\mathrm{b}^{2}+4|\mathrm{a}| \mathrm{c}}}{2|\mathrm{a}|}}$,

$\sqrt{\max \left(f^{2}\left(M_{\chi}\right), 2 \lambda_{\phi s} v_{2}^{2}-4 \pi v_{1}^{2}\right)}<M_{s}<\sqrt{2 \lambda_{\phi s} v_{2}^{2}+4 \pi v_{1}^{2}}$.

\section{Relic abundance}

The evolution of the number density of DM particles with time is governed by the Boltzmann equation. In this section, we compute the relic density for both DM candidates scalar and fermion in our model, at the present epoch. In general, the coupled Boltzmann equations for two-component DM $S$ and $\chi$ should be solved in order to compute the number density. The coupled Boltzmann equations for scalar $S$ and fermion $\chi$ are given by

$$
\begin{aligned}
\frac{\mathrm{d} n_{\chi}}{\mathrm{d} t}+3 H n_{\chi}= & -\sum_{j=p, H_{1}, H_{2}}\left\langle\sigma_{\chi \chi} \rightarrow j j v\right\rangle\left(n_{\chi}^{2}-n_{\chi, e q}^{2}\right) \\
& -\left\langle\sigma_{\chi \chi} \rightarrow S S v\right\rangle\left(n_{\chi}^{2}-n_{\chi, e q}^{2} \frac{n_{S}^{2}}{n_{S, e q}^{2}}\right), \\
\frac{\mathrm{d} n_{S}}{\mathrm{~d} t}+3 H n_{S}= & -\sum_{j=p, H_{1}, H_{2}}\left\langle\sigma_{S S \rightarrow j j} v\right\rangle\left(n_{S}^{2}-n_{S, e q}^{2}\right) \\
& -\left\langle\sigma_{S S \rightarrow \chi \chi} v\right\rangle\left(n_{S}^{2}-n_{S, e q}^{2} \frac{n_{\chi}^{2}}{n_{\chi, e q}^{2}}\right),
\end{aligned}
$$

where $p$ denotes any SM particles. In $\left\langle\sigma_{a b \rightarrow c d} v\right\rangle$ all annihi-

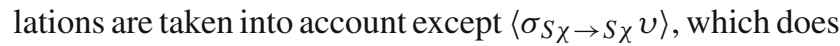
not affect the number density. By using $x=m / T$, where $T$ is the photon temperature, as the independent variable instead 
of time and $\dot{T}=-H T$, one can rewrite the Boltzmann equations in terms of the yield quantity, $Y=n / s$ :

$$
\begin{aligned}
\frac{\mathrm{d} Y_{\chi}}{\mathrm{d} x}= & -\sqrt{\frac{45}{\pi}} M_{p l} g_{*}^{1 / 2} \frac{m}{x^{2}}\left[\sum_{j=p, H_{1}, H_{2}}\left\langle\sigma_{\chi \chi \rightarrow j j} v\right\rangle\left(Y_{\chi}^{2}-Y_{\chi, e q}^{2}\right)\right. \\
& \left.+\left\langle\sigma_{\chi \chi} \rightarrow S S v\right\rangle\left(Y_{\chi}^{2}-Y_{\chi, e q}^{2} \frac{Y_{S}^{2}}{Y_{S, e q}^{2}}\right)\right] \\
\frac{\mathrm{d} Y_{S}}{\mathrm{~d} x}= & -\sqrt{\frac{45}{\pi}} M_{p l} g_{*}^{1 / 2} \frac{m}{x^{2}}\left[\sum_{j=p, H_{1}, H_{2}}\left\langle\sigma_{S S \rightarrow j j} v\right\rangle\left(Y_{S}^{2}-Y_{S, e q}^{2}\right)\right. \\
& \left.+\left\langle\sigma_{S S \rightarrow \chi \chi} v\right\rangle\left(Y_{S}^{2}-Y_{S, e q}^{2} \frac{Y_{\chi}^{2}}{Y_{\chi, e q}^{2}}\right)\right]
\end{aligned}
$$

where $M_{p l}$ is the Planck mass and $g_{*}^{1 / 2}$ is the effective numbers parameter. As seen in the above equations, there are new terms in the Boltzmann equations, which describe the conversion of two DM particles into each other, $\langle S S \leftrightarrow \chi \chi\rangle$. These two cross sections are also described by the same matrix element. Therefore, we expect that $\left\langle\sigma_{\chi x \rightarrow S S} v\right\rangle$ and $\left\langle\sigma_{S S \rightarrow \chi x} v\right\rangle$ are not independent and their relation is

$Y_{\chi, e q}^{2}\left\langle\sigma_{\chi \chi} \rightarrow S S v\right\rangle=Y_{S, e q}^{2}\left\langle\sigma_{S S \rightarrow \chi \chi} v\right\rangle$.

The interactions between the two DM components take place by exchanging two scalar mass eigenstates $H_{1}$ and $H_{2}$ where the coupling of $\chi$ to $H_{1}$ is suppressed by $\sin \alpha$. Therefore, it usually is the $\mathrm{H}_{2}$-mediated diagram that gives the dominant contribution. However, if one DM particle is heavier than the other one, (16), the conversion of the heavier particle into the lighter one is relevant, $S S \rightarrow \chi \chi$. Thus, the contribution of $\chi$ to the relic density is dominant and the only option for annihilation of $\chi$ is via $H_{1}$-mediated and $\mathrm{H}_{2}$-mediated diagrams into SM particles.

To solve numerically the two coupled Boltzmann differential equations, we have implemented the model into micrOMEGAs [36] (via LanHEP [37]). Since we have two stable DM particles, the DM constraint in this model reads

$\Omega_{D M} h^{2}=\Omega_{S} h^{2}+\Omega_{\chi} h^{2}=0.1199 \pm 0.0027$

according to the data by the Planck collaboration [38]. Another related quantity is the fraction of the DM density that is due to $S$ and $\chi$, denoted by $\xi_{S}$ and $\xi_{\chi}$, respectively. So

$\xi_{\chi}=\frac{\Omega_{\chi}}{\Omega_{D M}}, \quad \xi_{S}=\frac{\Omega_{S}}{\Omega_{D M}}, \quad$ with $\xi_{\chi}+\xi_{S}=1$.

Figures 1, 2, 3, and 4 depict the relic density of fermionic and scalar DM as a function of the DM mass. According to these plots, the highest contribution of DM relic density $\Omega_{D M}$ comes from fermionic DM, i.e., $\Omega_{\chi}$. Since in our model

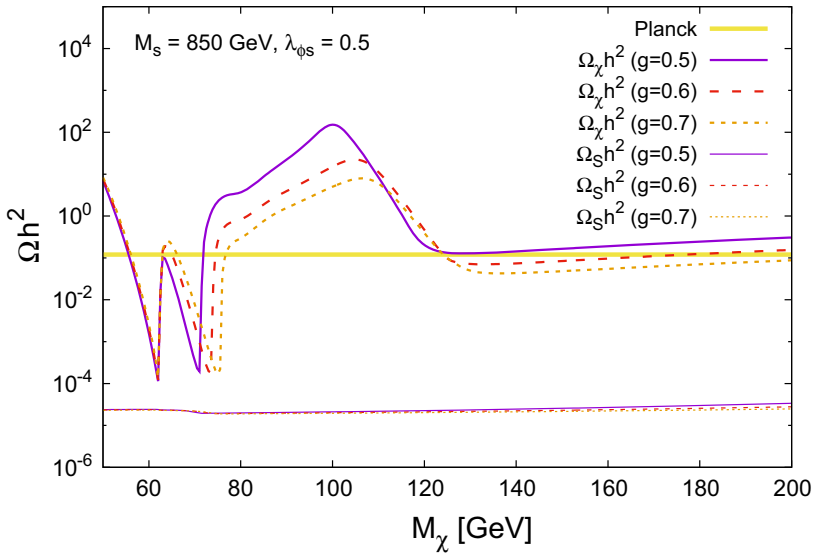

Fig. 1 Relic density as a function of fermionic DM mass for different values of the coupling $g$

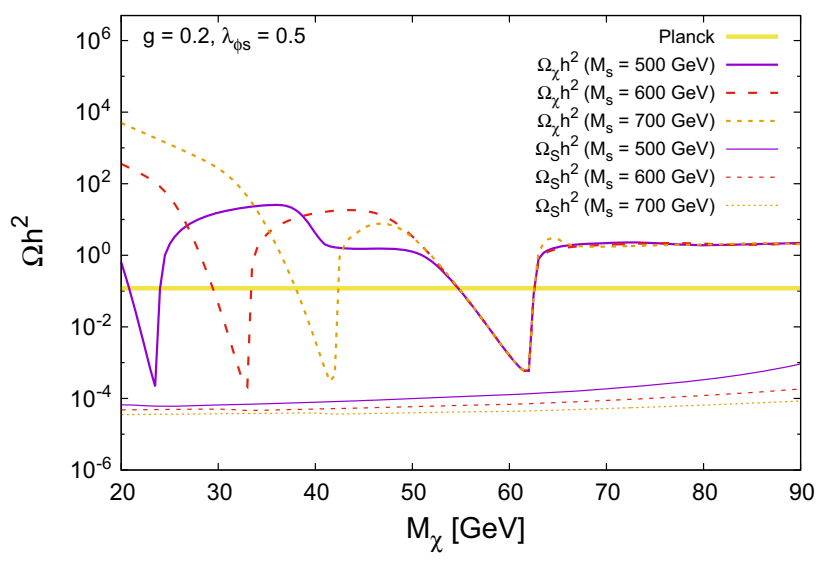

Fig. 2 Relic density as a function of fermionic DM mass for different values of the scalar mass $M_{s}$

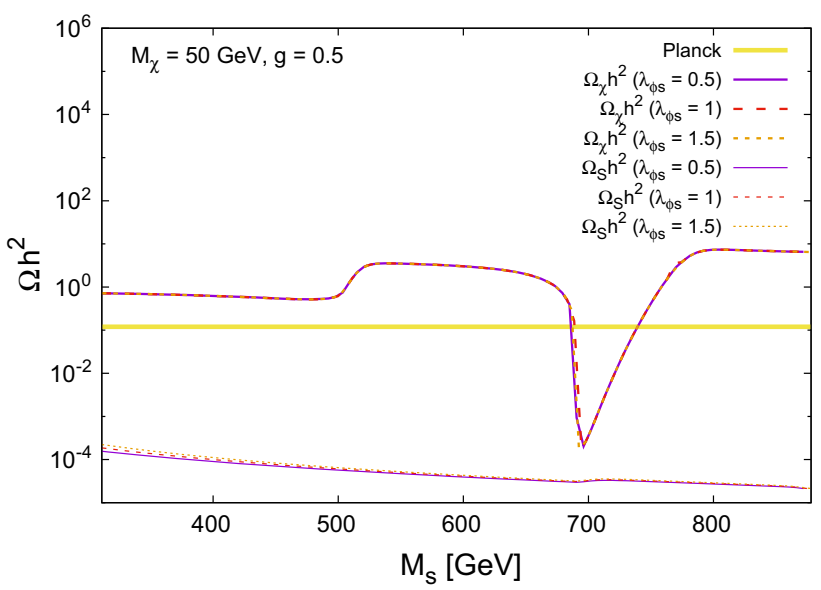

Fig. 3 The relic density as a function of the scalar DM mass for different values of coupling $\lambda_{\phi s}$

scalar DM is always heavier than fermionic DM, in addition to annihilation to SM particles, it could also annihilate to 


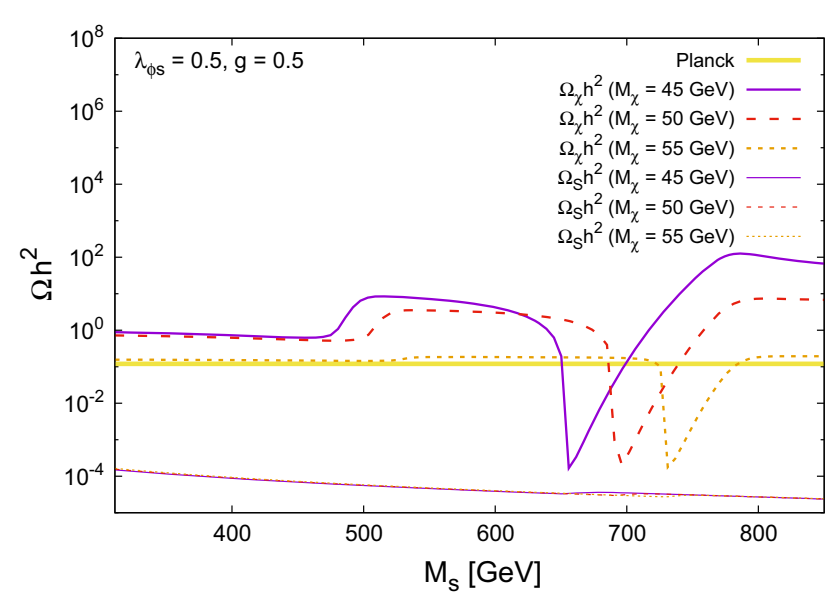

Fig. 4 Relic density as a function of the scalar DM mass for different values of the fermionic mass $M_{\chi}$

fermionic DM particles. Therefore, its relic density is smaller than the fermionic relic density.

Figure 1 shows the two DM relic densities as a function of $M_{\chi}$ for different values of $g$. For any given value of $g$ the fermionic relic density $\Omega_{\chi}$ features a double reduction at the $H_{1}$ and $H_{2}$ resonances (respectively, at $M_{\chi}=\frac{M_{H_{1}}}{2}=$ $62.5 \mathrm{GeV}$ and at $M_{\chi}=\frac{M_{\mathrm{H}_{2}}}{2}$ ). There is another reduction due to the opening of the $\chi \chi \rightarrow \mathrm{H}_{2} \mathrm{H}_{2}$ annihilation channel. Note that, according to Eq. (14), $M_{\mathrm{H}_{2}}$ itself depends on $g$, $M_{s}$ and $M_{\chi}$, so it is not an independent parameter. Therefore, in our relic density plots, it varies with $g$ and DM masses. In Fig. 1, scalar relic density $\Omega_{S}$ does not vary dramatically with $M_{\chi}$ or $g$. Note that $\lambda_{s H}$ is a determinative parameter in scalar DM annihilation to SM particles. On the other hand, annihilation of scalar DM to SM particles is more favorable than its annihilation to fermionic DM, because most SM particles are lighter than fermionic DM. Therefore, $\Omega_{S}$ mostly depends on $\lambda_{s H}$, rather than $\lambda_{\phi s}$. According to Eqs. (13) $\lambda_{s H}$ is given by

$\lambda_{s H}=\frac{M_{s}^{2}}{v_{1}^{2}}-\frac{2 \lambda_{\phi s} M_{\chi}^{2}}{g^{2} v_{1}^{2}}$,

and for the given parameters in Fig. $1, \lambda_{s H}$ is mostly determined by the first term of Eq. (42). Thus, it does not vary much with $M_{\chi}$ or $g$.

In Fig. 2 DM relic densities are plotted versus $M_{\chi}$ for different values of $M_{s}$. Similarly, for the given values of $M_{s}$ the fermionic relic density again features a double reduction at the $H_{2}$ and $H_{1}$ resonances (respectively, at $M_{\chi}=\frac{M_{H_{2}}}{2}$ and $M_{\chi}=\frac{M_{H_{1}}}{2}=62.5 \mathrm{GeV}$ ). Obviously, in this plot $M_{H_{2}}$ at the first resonance is lighter than $M_{H_{1}}=125 \mathrm{GeV}$. For the scalar relic density, according to Eq. (42), a larger $M_{s}$ leads to a larger $\lambda_{s H}$ and therefore the DM-SM interaction gets stronger, which leads to a smaller scalar relic density. Furthermore, now for $M_{s}=500 \mathrm{GeV}$, the second term of Eq. (42) can compete with the first term, and with the growth of $M_{\chi}, \lambda_{s H}$ will decrease. Due to this reduction, the scalar DMSM interaction becomes weaker and therefore $\Omega_{S}$ increases with $M_{\chi}$. For larger $M_{s}$ (for example $M_{s}=700 \mathrm{GeV}$ again the first term of Eq. (42) dominates and $\Omega_{S}$ increases less with $M_{\chi}$.

Figures 3 and 4 depict relic densities versus $M_{s}$. In Fig. 3, for $M_{\chi}=50 \mathrm{GeV}$ there is a single reduction in fermionic relic density around $M_{s}=700 \mathrm{GeV}$. This reduction corresponds to $M_{H_{2}}=2 M_{\chi}=100 \mathrm{GeV}$ which is a resonance case. According to Eq. (42), $\lambda_{s H}$ increases with $M_{S}$ and the scalar DM-SM interaction becomes stronger. Therefore, $\Omega_{S}$ decreases with $M_{S}$. In addition, for the given parameters, since the first term of Eq. (42) dominates, $\lambda_{s H}$, and therefore $\Omega_{S}$, is nearly independent of $\lambda_{\phi s}$. In this figure, only for small $M_{s}$ a little dependency of $\Omega_{S}$ to $\lambda_{\phi s}$ can be realized.

Finally, in Fig. 4 we display the fermionic relic density as a function of $M_{S}$ for different values of $M_{\chi}$. Therefore, we have different resonance cases, corresponding to $M_{H_{2}}=2 M_{\chi}$ for each value of $M_{\chi}$. For the given parameters, the scalar relic density is not sensitive to different values of $M_{\chi}$, because as mentioned before $\Omega_{S}$ is mostly determined by $\lambda_{s H}$, and, again according to Eq. (42), the second term can be neglected in comparison with the first term. Thus, for the given values of Fig. 4, only the first term, which is independent of $M_{\chi}$, affects the scalar DM relic density so that on growing $M_{S}$, $\lambda_{S H}$ increases and consequently $\Omega_{S}$ decreases.

In our model, the total DM relic density does not depend on $\lambda_{\phi s}$. This parameter can only affect $\Omega_{S}$, which has a small contribution in $\Omega_{D M}=\Omega_{S}+\Omega_{\chi}$. Therefore, $\Omega_{D M}$ only depends on $g, M_{s}$, and $M_{\chi}$.

\section{Phenomenological aspects}

\subsection{Direct detection}

In this section, we investigate constraints on the parameter space of our model which are imposed by the search for scattering of DM-nuclei. Since no such collision events have been observed yet by different DM direct detection experiments, these experiments provide an exclusion limit on the DM-nucleon scattering cross section. The strongest bounds on the DM-nucleon cross section have been obtained by the XENON1T [39] and LUX [40] experiments:

XENON1T : $\sigma_{\mathrm{SI}} \leq 4.1 \times 10^{-47} \mathrm{~cm}^{2}$,

$$
\text { LUX : } \sigma_{\mathrm{SI}} \leq 2.2 \times 10^{-46} \mathrm{~cm}^{2} \text {. }
$$

The spin-independent direct detection cross section of $\chi$ is determined by $H_{1}$ and $H_{2}$ exchanged diagrams: 


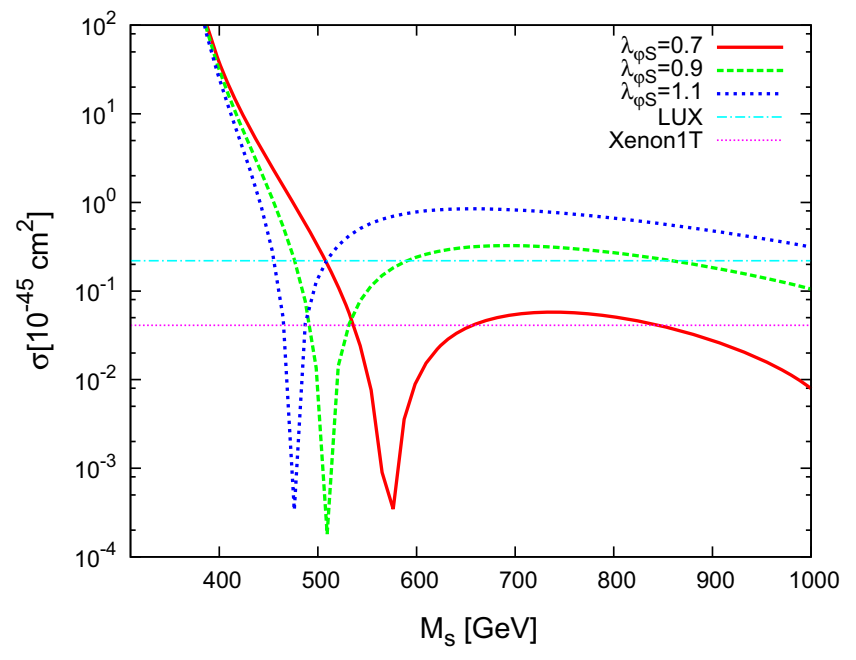

(a)

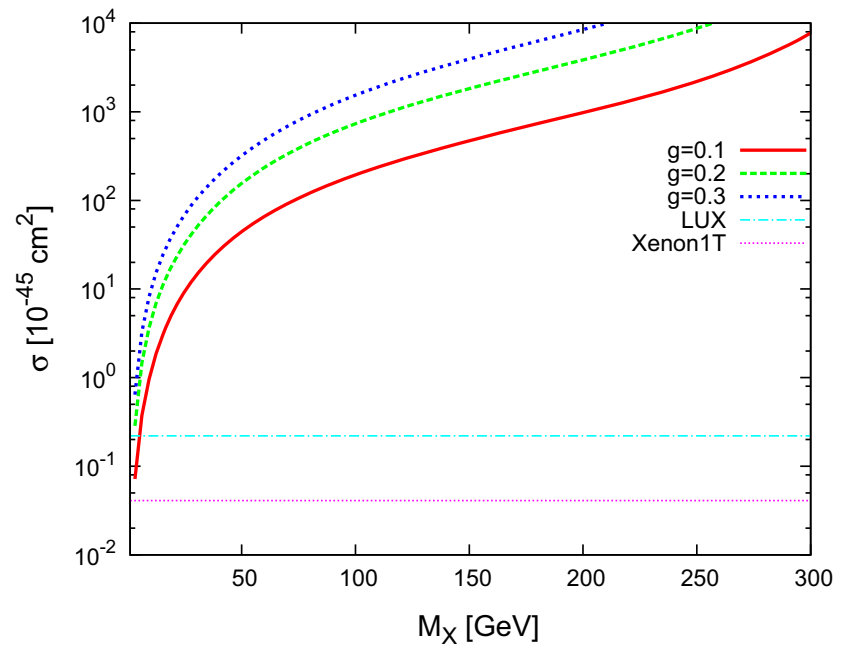

(b)

Fig. 5 The direct detection cross section as a function of mass of a scalar DM. We set $M_{\chi}=200 \mathrm{GeV}$ and $g=0.2$. b Fermion DM. We set $M_{s}=500 \mathrm{GeV}$

$\sigma_{\chi}=\xi_{\chi} \frac{g^{3} v_{1}}{\pi M_{\chi}\left(1+\left(v_{1} g / M_{\chi}\right)^{2}\right)} \mu_{\chi}^{2}\left(\frac{1}{m_{H_{1}}^{2}}-\frac{1}{m_{H_{2}}^{2}}\right)^{2} f_{n}^{2}$

where $\xi_{\chi}=\frac{\Omega_{\chi}}{\Omega_{D M}}$ and $\mu_{\chi}$ is the reduced mass of nucleon and fermionic DM and the coupling constant $f_{n}$ is given by nuclear matrix elements and nucleon mass [41]. Similarly, for the scalar DM candidate the effective spin-independent direct detection cross section is given by

$$
\begin{aligned}
\sigma_{S}= & \xi_{S} \frac{\mu_{S}^{2}}{4 \pi M_{H_{1}}^{4} M_{H_{2}}^{4} m_{S}^{2}} \\
& {\left[\frac{M_{s}^{2}-2 \lambda_{\phi s} M_{\chi}^{2} / g^{2}}{v_{1}}\left(\frac{M_{H_{2}}^{2}}{1+\left(v_{1} g / M_{\chi}\right)^{2}}+\frac{M_{H_{1}}^{2} g^{2} v_{1}^{2}}{g^{2} v_{1}^{2}+M_{\chi}^{2}}\right)\right.} \\
& \left.+\frac{2 v_{1} \lambda_{\phi s}}{1+\left(v_{1} g / M_{\chi}\right)^{2}}\left(M_{H_{1}}^{2}-M_{H_{2}}^{2}\right)\right]^{2} f_{n}^{2}
\end{aligned}
$$

where $\xi_{S}=\frac{\Omega_{S}}{\Omega_{D M}}$ and $\mu_{S}$ are the reduced masses of nucleon and scalar DM. The parameters $\lambda_{\phi s}$ and $g$ are independent and have been defined in the previous section. It is remarkable that the two terms in Eq. (44) may cancel against each other, giving a suppressed cross section. In Fig. 5, we display the direct detection cross section as a function of the mass of scalar and fermion DM. As seen in Fig. 5a, $\sigma_{S}$ has a minimum in the value of $M_{S}$ at which cancellation takes place. For scalar DM, the direct detection cross section depends on the scalar DM mass, $\lambda_{\phi s}, g$ and $M_{\chi}$. The fermionic DM direct detection cross section does not depend on $\lambda_{\phi s}$. However, as mentioned in the previous section, $m_{\mathrm{H}_{2}}$ is not an independent parameter and depends on three independent parameters of our model, $M_{s}, g$ and $M_{\chi}$. Also $m_{H_{2}}$ may be very small and so the contribution of its propagator to the direct detection cross section can be very large. For this reason, a large portion of parameter space is excluded by this observable. In order to show the allowed region in parameter space, we display scatter points in Fig. 6. Figure $6 a-c$ depict the allowed regions in $g, \lambda_{\phi s}$ and $M_{s}$ for scalar DM and Fig. 6d depicts the allowed regions in $g$ and $M_{\chi}$ for fermionic DM, which are consistent with experimental measurements of $\sigma_{\text {Xenon100 }}$ and $\sigma_{\text {LUXX }}$.

Notice that in the above analysis we separately suppose $\xi_{S}=1$ and $\xi_{\chi}=1$ in Fig. 5a, b. In the next step, we display a combined analysis, direct detection and relic density in Fig. 7. In order to study the effect of the direct detection experiment on the model, the rescaled DM-nucleon cross section $\xi_{\chi} \sigma_{\chi}$ and $\xi_{S} \sigma_{S}$ should be considered. Scatter points in Fig. 7 (left) show the allowed region in parameter space of the model in the $M_{s}$ and $M_{\chi}$ plane for different parameters of the model which are consistent with the observed relic density by the Planck collaboration [38]. In these figures, it is supposed that $0.11<\Omega h^{2}<0.13$ for the allowed range of relic density and also $0<\lambda_{\phi s}<3$, and $0.5<$ $g<1.5$. The right figures depict the rescaled DM-nucleon cross section versus DM mass for different values of the other model parameters. The solid line determines the upper limit of the LUX experiments [40] for the direct detection of DM and the dashed line shows the regions of parameter space which are expected to be probed by the future direct detection experiment XENONnT [42]. 


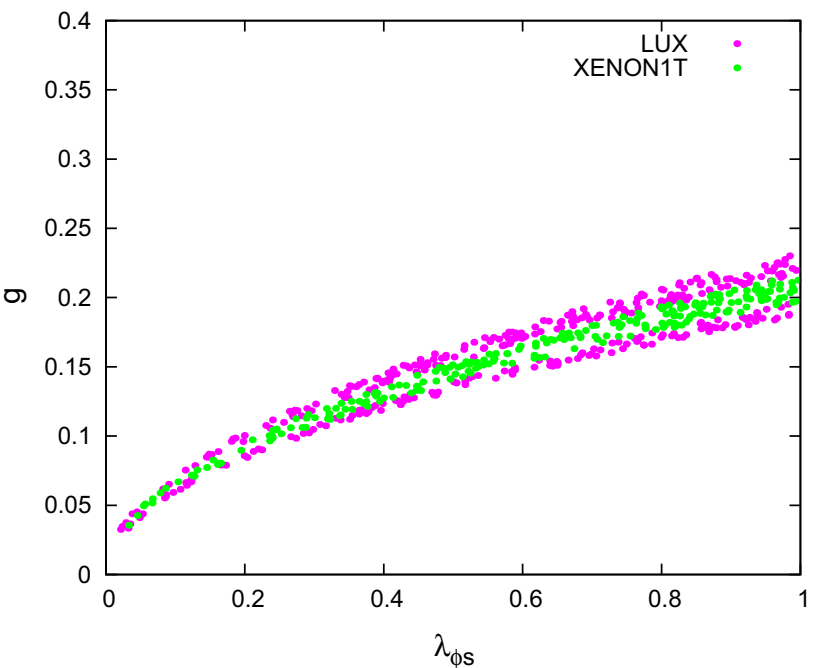

(a)

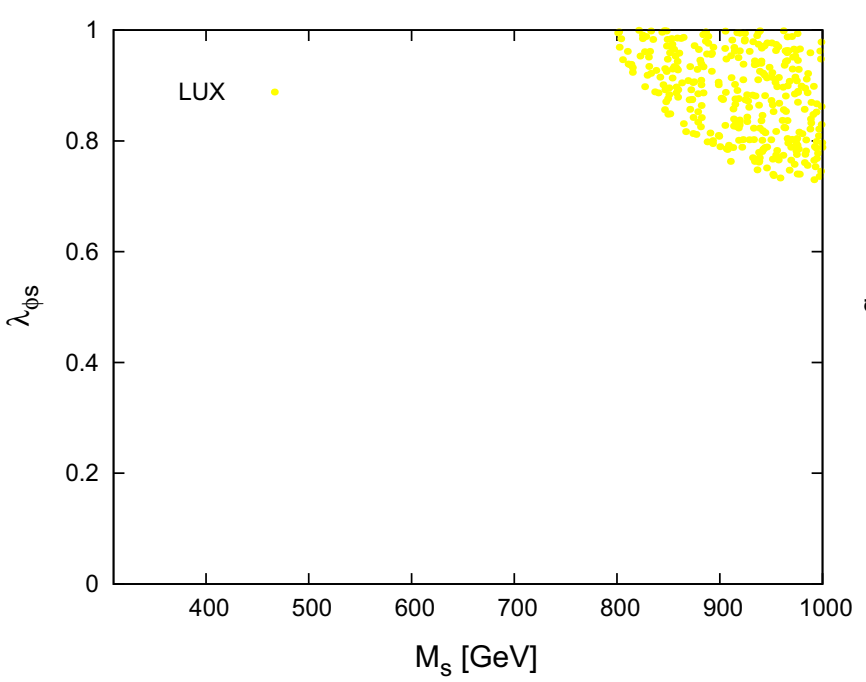

(c)

Fig. 6 a-c Depict the ranges of parameter space in the $g, \lambda_{\phi s}$ and $M_{s}$ planes for scalar DM and $\mathbf{d}$ depicts the allowed regions in $g$ and $M_{\chi}$ for fermionic DM, which are consistent with experimental measurements

\subsection{Indirect detection}

The indirect detection of DM annihilation and decay using observations of photons, charged cosmic rays, and neutrinos offers a promising means of identifying the nature of this part of the Universe. There are currently intensive international efforts to detect these astroparticles as signature of DM particles.

Indirect detection experiments hunt for the selfannihilation (or decay) products of DM particles in regions of high DM density (e.g., the center of our galaxy). Two dark matter particles could annihilate to produce gamma rays or SM particle-antiparticle pairs. Indirect detection experi-

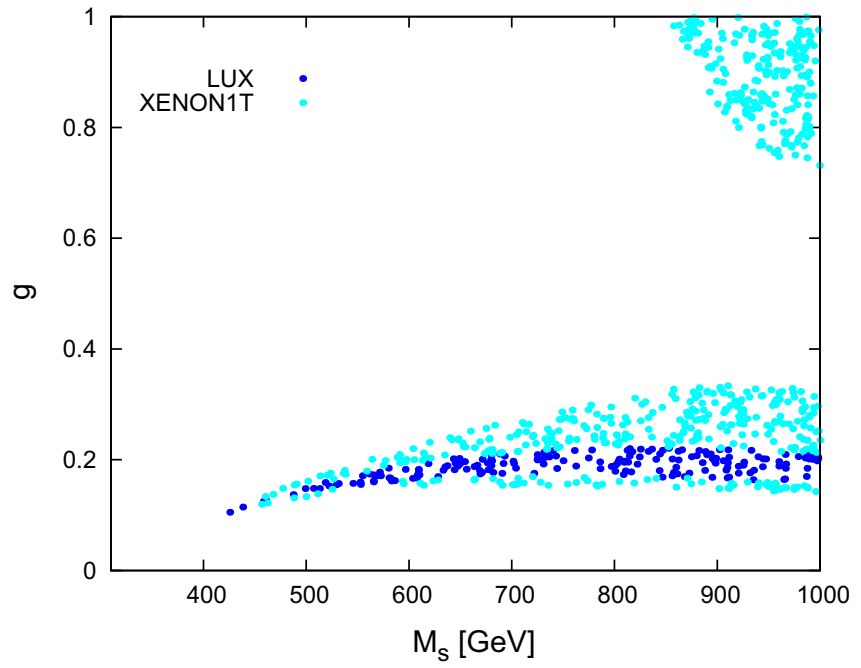

(b)

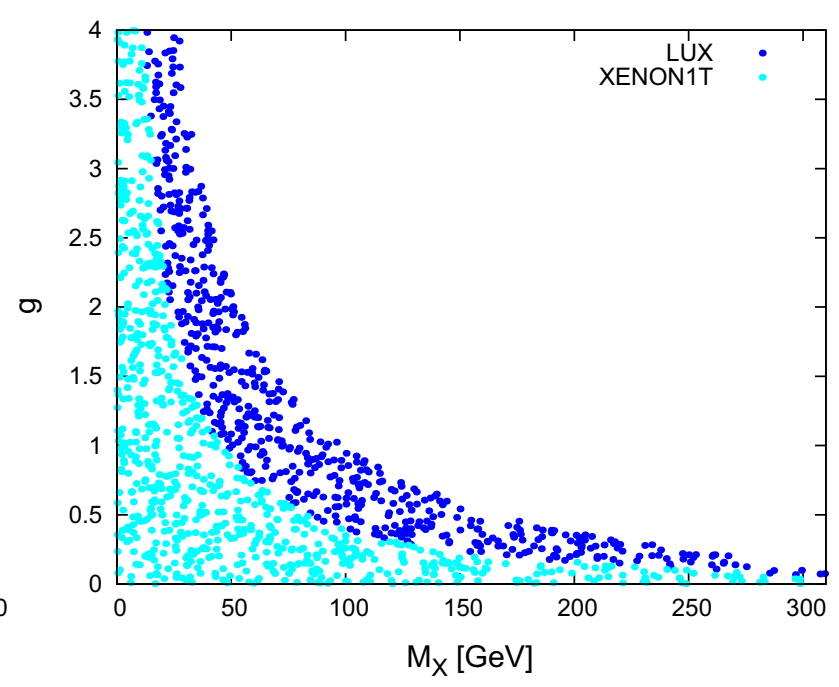

(d)

of $\sigma_{\text {Xenon1T }}$ and $\sigma_{\text {LUX }}$. In a, we set $M_{s}=500 \mathrm{GeV}$ and $M_{\chi}=200 \mathrm{GeV}$. In b, we set $M_{\chi}=200 \mathrm{GeV}$ and $g=0.5$. In d, we set $M_{s}=500 \mathrm{GeV}$

ments may confirm DM annihilation through an excess of gamma rays (e.g., the Fermi-LAT experiment [43]), positrons (e.g., the PAMELA experiment [44]), antiprotons (e.g., the AMS experiment [45]) or neutrinos (e.g., the IceCube experiment [46]). A major difficulty is that various astrophysical sources can resemble closely the signal expected from DM. Therefore, multiple signals are required for a conclusive discovery.

In the freeze-out scenario, the pair annihilation rate of a thermal relic DM particle is directly linked to today's relic abundance. Based on the measured abundance of DM, a particle which constitutes all of the DM will have a total pair annihilation cross section of $\langle\sigma v\rangle \sim O\left(10^{-26}\right) \mathrm{cm}^{3} / \mathrm{s}$ 

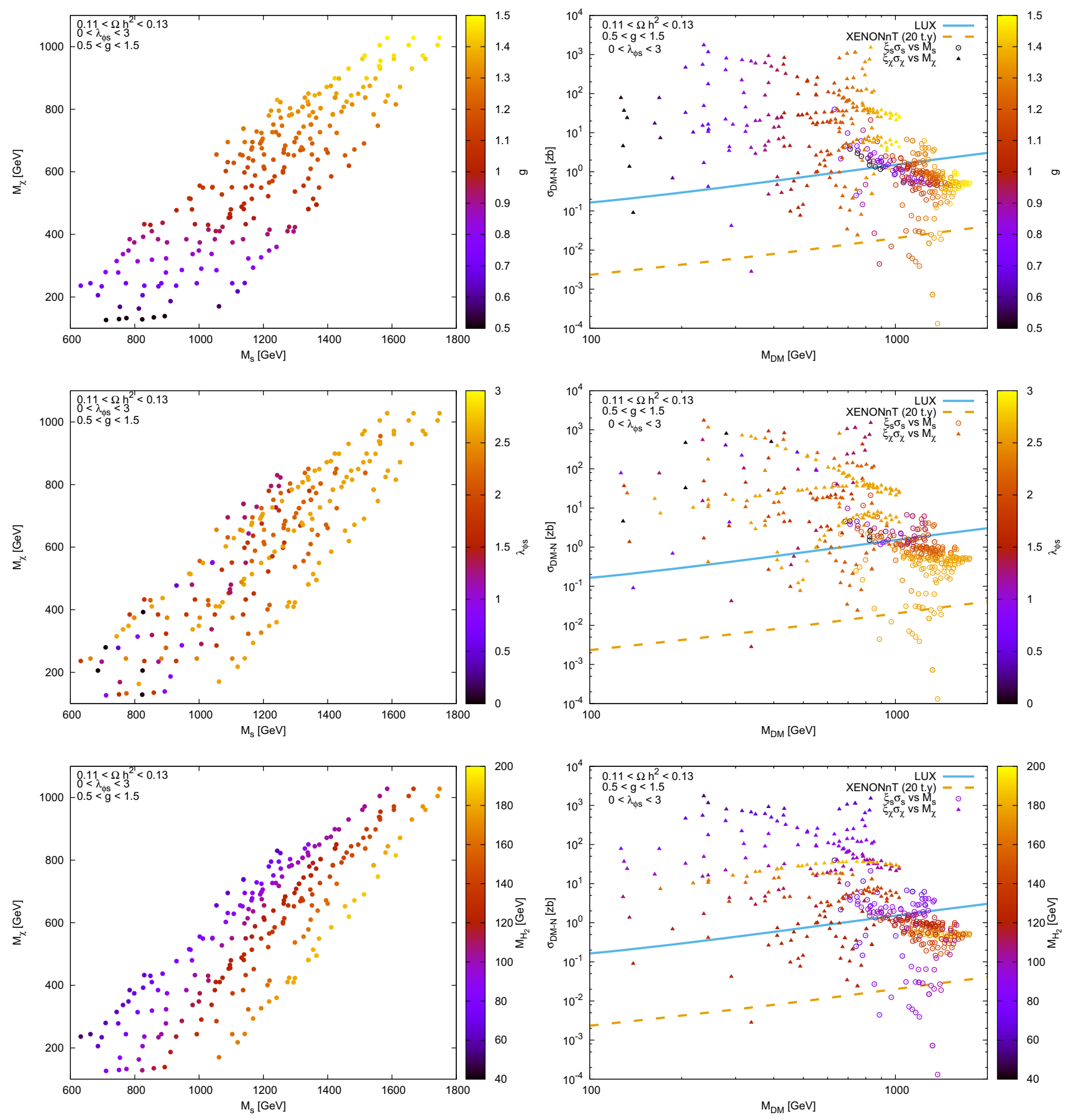

Fig. 7 (Left) Scatter points depict the ranges of the parameter space of the model in $M_{s}$ and $M_{\chi}$ plane for different parameters of the model, which are consistent with the observed relic density by the Planck collaboration [38]. (Right) Rescaled DM-nucleon cross section as a func-

tion of the DM mass for different values of the other model parameters. One can also see LUX' upper bound [40] and the regions of parameter space which are expected to be probed by the future direct detection experiment XENONnT [42]

[48]. This value is often used as a benchmark and is referred to as the thermal relic cross section. Indirect detection experiments which search for gamma rays and cosmic rays have recently become sensitive to dark matter with this benchmark cross section for masses up to around the weak scale, $\mathrm{O}(100$

$\mathrm{GeV})$. We have calculated the velocity-averaged annihilation cross section of DM for $0.11<\Omega h^{2}<0.13,0<\lambda_{\phi s}<3$, and $0.5<g<1.5$ by using the micrOMEGAs package [36]. Our result is shown in Fig. 8. As is seen, the results cannot saturate the particle fluxes detected in the aforementioned 


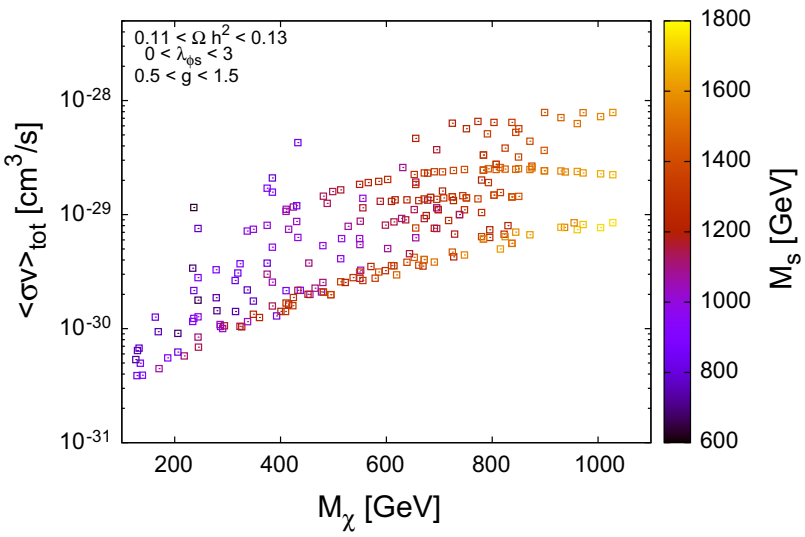

Fig. 8 Dependence of velocity-averaged annihilation cross section on DM mass

indirect detection experiments limits. Figure 8 shows that the annihilation cross section is always less than two orders of magnitude smaller than the expected thermal cross section. For exploring the DM properties in indirect detection experiments, large annihilation rates are desirable. The DM annihilation rate will usually be largest if it proceeds via an $\mathrm{s}$-wave process. Unfortunately, for the fermionic component, which dominates the relic density, the s-wave annihilation to $\mathrm{SM}$ products is absent. Therefore, the annihilation cross section of our model is not large enough to give a signal in indirect detection experiments, as the p-wave term is suppressed by a factor of the DM velocity squared (with $V_{\mathrm{DM}} \sim 10^{-3}$ for dark matter particles in the galactic halo). This means that astroparticle fluxes which are coming from the galactic center could have other astrophysical origins.

\subsection{Self-interaction}

The self-interaction of DM can potentially be probed by studying the offset between the DM halo and the stars of a galaxy moving through a region of large DM density. The first evidence for DM self-interactions has been reported [49] based on observations of four elliptical galaxies in the inner $10 \mathrm{kpc}$ core of the galaxy cluster Abell 3827. An updated work [50] has considered a set of 12 galaxies and six clusters in order to cover different scales. Including the core sizes from dwarf to cluster (varying from 0.5 to $50 \mathrm{kpc}$ ), the aforementioned cross section is parametrized as

$\sigma_{\text {self }}^{\text {eff }} / m_{\text {DM }} \sim 0.1-2 \mathrm{~cm}^{2} \mathrm{~g}^{-1}$

where the effective self-interacting cross section is defined by $\sigma_{\text {self }}^{\text {eff }} / m_{\mathrm{DM}}=\xi_{\chi, S}^{2} \frac{\sigma_{\text {self }}}{m_{\mathrm{DM}}}$ and $\xi_{\chi, S}$ is the fraction of one of the two DM components. In particle physics units, this corresponds to $\sigma_{\text {self }}^{\text {eff }} / m_{\mathrm{DM}} \sim(0.43-8.72) \times 10^{3} \mathrm{GeV}^{-3}$.

In the next step, we consider the DM self-interacting cross section for scalar $S$ and fermion $\chi$ DM. The DM selfinteractions include the processes $S S \longrightarrow S S, \chi \chi \longrightarrow \chi \chi$, $S S \longrightarrow \chi \chi, \chi \chi \longrightarrow S S$ and $S \chi \longrightarrow S \chi$. Figure 9 shows Feynman diagrams for DM self-interactions.

The main contributions to $\sigma / M_{s}$ for scalar annihilation (processes $S S \longrightarrow S S[51]$ and $S S \longrightarrow \chi \chi$ ) are given in appendix. For the process $S S \longrightarrow S S[51], \sigma / M_{S}$ is proportional to $1 / M_{s}^{3}$ and after imposing the constraint $M_{s}>$ $310 \mathrm{GeV}$, we find that this situation does not saturate the upper bound on the self-interaction cross section. Indeed, to obtain a reasonably strong scalar DM self-interaction, the mass of the scalar must be very small, $M_{s}<1 \mathrm{GeV}$. Since in the non-relativistic regime $s \sim 4 M_{s}^{2}, \sigma(S S \rightarrow \chi \chi) / M_{s}$ will be larger than $\sigma(S S \rightarrow S S) / M_{s}$, This feature is depicted in Fig. 10a. As is seen in this figure, the self-interaction for scalar DM is very much smaller than the upper bound. However, it is possible to obtain an upper bound on the selfinteraction cross section for scalar DM if we consider selfinteraction in the vicinity of resonance $M_{s} \simeq M_{H_{2}} / 2$. Note that according to Eq. (15), the mass of scalar DM cannot be
Fig. 9 The Feynman diagrams for scalar and fermion DM self-interactions
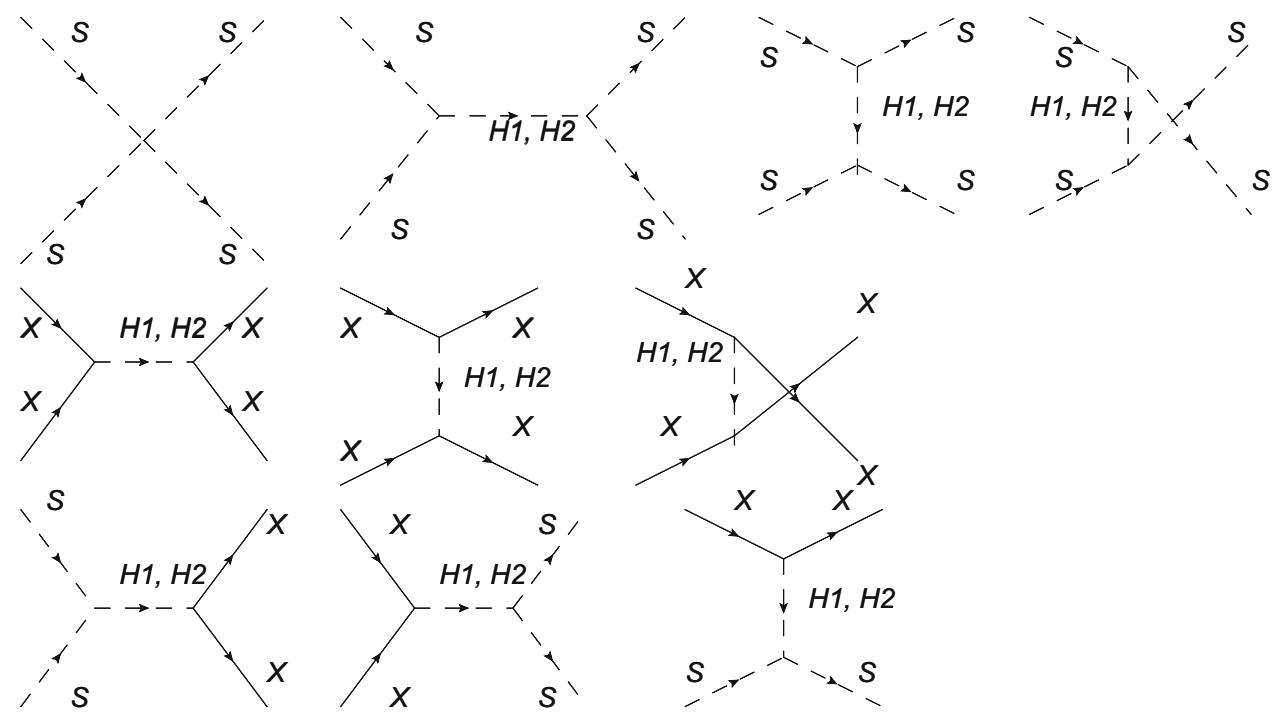

$S^{\prime}$

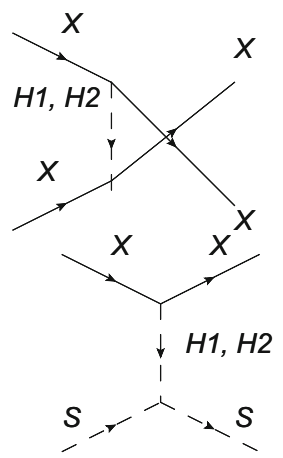




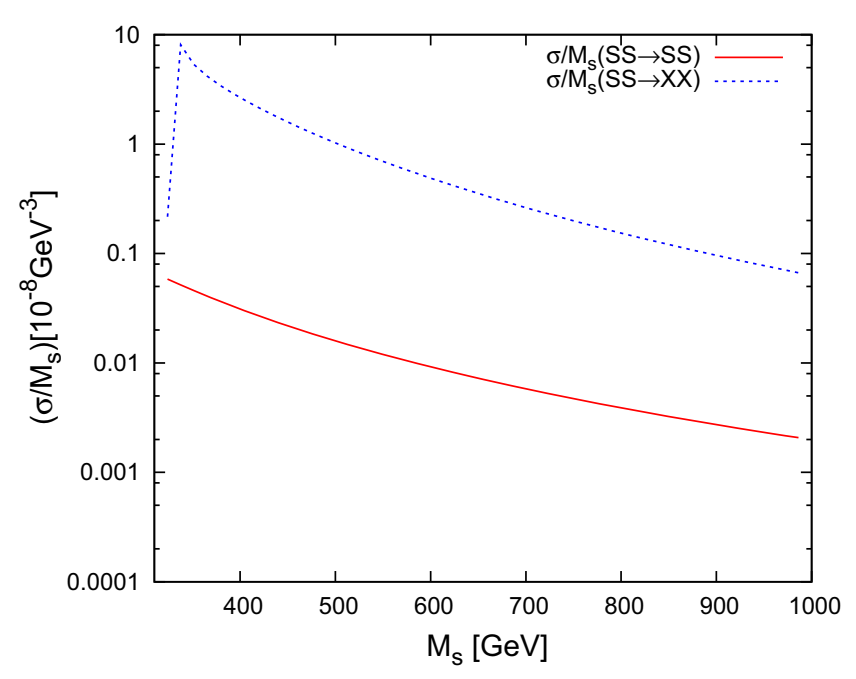

(a)

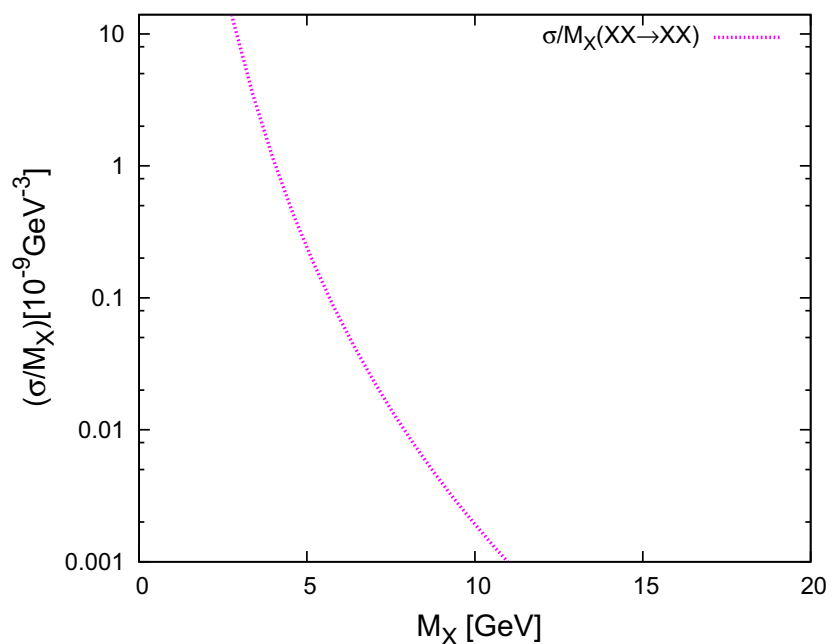

(b)

Fig. 10 a The scalar self-interaction cross section as a function of the scalar DM mass. We set $M_{\chi}=100 \mathrm{GeV}$ and $g=0.5 \lambda_{\phi s}=2$ and $\lambda_{s}=2$. b The fermionic self-interaction cross section as a function of the fermion DM mass. The input parameters are similar to (a) except $M_{s}=500 \mathrm{GeV}$

equal to half of the SM Higgs mass. For the resonance regime $\left(M_{s} \simeq M_{H_{2}} / 2\right)$, the s-channel $H_{2}$ exchange diagram in Fig. 9 dominates and the scalar DM self-interaction may exceed the experimental bound. Achieving the observed scalar DM selfinteraction cross section requires that $M_{s}$ be severely tuned such that $\left|M_{S}-M_{H_{2}} / 2\right|<1 \mathrm{MeV}$ (while $M_{s}>310 \mathrm{GeV}$ ). However, since the main contribution of the observed relic density was obtained from fermionic DM, and scalar DM has a small contribution to the relic density, we expect that this process is very rare in the center of the Milky Way.

In the following, we consider the self-interaction for the case of Dirac fermionic DM which includes the processes $\chi \chi \longrightarrow \chi \chi$ and $\chi \chi \longrightarrow S S$. The main Feynman diagrams which contribute to the aforementioned process are of s-channel type for $\chi \chi \longrightarrow \chi \chi$ in Fig. 9 and $\chi \chi \longrightarrow S S$. The cross sections of these processes are presented in the appendix.

For the process $\chi \chi \longrightarrow S S$ in the non-relativistic limit $s<4 M_{s}^{2}$ and so this process is forbidden. For the processes $\chi \chi \longrightarrow \chi \chi$, since in the non-relativistic regime $s \simeq 4 M_{\chi}^{2}$, the self-interaction of fermionic DM is much smaller than the experimental bound (it is shown in Fig. 10b). It also turns out that to bring about a reasonably strong fermionic DM selfinteraction (similar to scalar DM), we should consider selfinteraction in the near resonance $M_{\chi} \simeq M_{H_{2}} / 2$ or $M_{H_{1}} / 2$. Notice that for fermionic DM, fine tuning should be stronger than for scalar DM due to the smaller self-interacting cross section for fermionic DM.

To continue, we also calculate the DM self-interaction cross sections for processes $S \chi \longrightarrow S \chi$ in non-relativistic limit. The cross sections is given in appendix. Given the fact that the main contribution of observed relic density

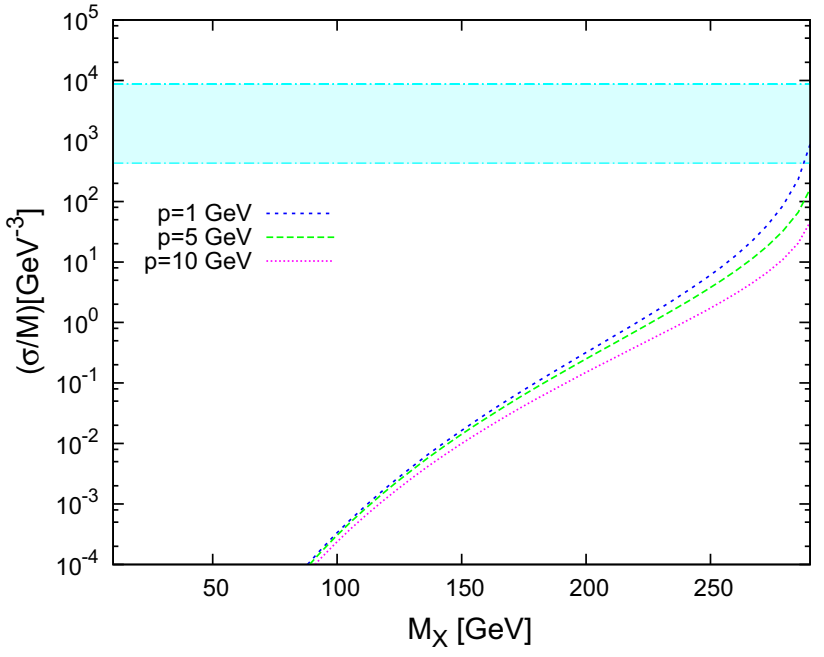

Fig. 11 The scalar-fermion self-interaction cross section as a function of the fermion DM mass. We set $M_{s}=500 \mathrm{GeV}$ and $g=0.5 \lambda_{\phi s}=$ $0.5, \lambda_{s}=0.5$ and different values for the momentum of the initial fermionic DM. The shadowed panel indicates the allowed range of the experimental measurements for the DM self-interaction

was obtained from fermionic DM and contribution of the scalar DM is less than 1 percent of total relic density, occurrence of this process is very rare. To estimate the magnitude of $\sigma_{S \chi} \longrightarrow S \chi$ in the non-relativistic limit, we suppose $s \simeq\left(M_{\chi}+M_{s}\right)^{2}$ and also consider $\bar{M}=\frac{\left(M_{s}+M_{\chi}\right)}{2}$. Note that this process does not affect the relic density of DM. In Fig. 11, we depict the contribution of $S \chi \longrightarrow S \chi$ versus $\bar{M}$ for several values of initial momentum of fermionic DM. As is seen, the specified process does not contribute to this cosmological constraint. In this estimation, we did not consider the difference in the fraction of the two DM components. 


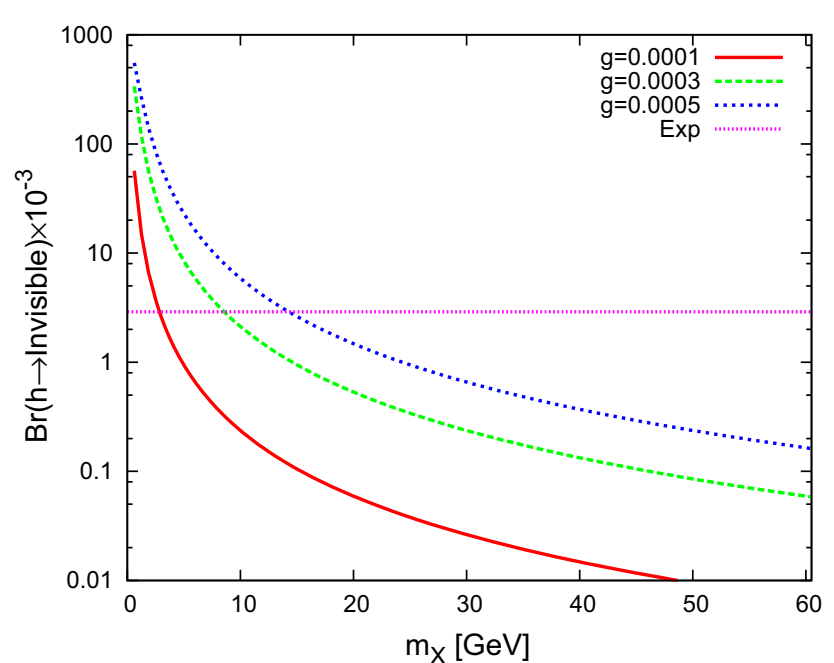

(a)

Fig. 12 a $\operatorname{Br}\left(H_{1} \rightarrow\right.$ Invisible $)$ as a function of fermionic DM mass for different values of $g$ coupling and $M_{s}=500 \mathrm{GeV}$. b Scatter points depict the ranges of parameter space in the mass of fermionic DM and

\subsection{Invisible Higgs decay}

The observed Higgs boson at $125 \mathrm{GeV}$ might decay to a component of DM which does not interact with the detector. Therefore it opens a window for exploring possible DMHiggs boson coupling. Notice that invisible Higgs boson decays are only sensitive to the DM coupling in the regions of parameter space which are kinematically allowed. Here, we suppose that $H_{1}$ is the SM Higgs boson; as a result, if scalon, scalar and fermionic DM are lighter than the SM Higgs boson, they can contribute to the invisible decay mode of the Higgs boson with branching ratio

$$
\begin{aligned}
\operatorname{Br} & \left(H_{1} \rightarrow \text { Invisible }\right) \\
& =\frac{\Gamma\left(\mathrm{H}_{1} \rightarrow \chi \chi\right)+\Gamma\left(\mathrm{H}_{1} \rightarrow \mathrm{SS}\right)+\Gamma\left(\mathrm{H}_{1} \rightarrow \mathrm{H}_{2} \mathrm{H}_{2}\right)}{\Gamma(\mathrm{h})_{\mathrm{SM}}+\Gamma\left(\mathrm{H}_{1} \rightarrow \mathrm{SS}\right)+\Gamma\left(\mathrm{H}_{1} \rightarrow \chi \chi\right)+\Gamma\left(\mathrm{H}_{1} \rightarrow \mathrm{H}_{2} \mathrm{H}_{2}\right)},
\end{aligned}
$$

where $\Gamma(h)_{S M}=4.15[\mathrm{MeV}]$ is the total width of the Higgs boson [52]. The decay rates for $H_{1} \rightarrow \chi \chi, H_{1} \rightarrow S S$ and $H_{1} \rightarrow H_{2} H_{2}$ are presented in the appendix. The branching ratio of the invisible Higgs mode has been constrained by various groups using the latest data from LHC [53-55]. The ATLAS collaboration has reported a search of the SM Higgs boson decay in its invisible decay mode, obtaining an upper limit of $75 \%$, at a mass of $125.5 \mathrm{GeV}$ [55]. In the SM, the main process which contributes to invisible decay of the Higgs boson is $h \rightarrow Z Z^{*} \rightarrow 4 v$, but $\mathrm{Br}\left(h \rightarrow Z Z^{*} \rightarrow 4 v\right)=$ $1.2 \times 10^{-3}$ [56] is below the sensitivity of the ATLAS collaboration analysis. According to Eq. (15), $M_{S}>310 \mathrm{GeV}$

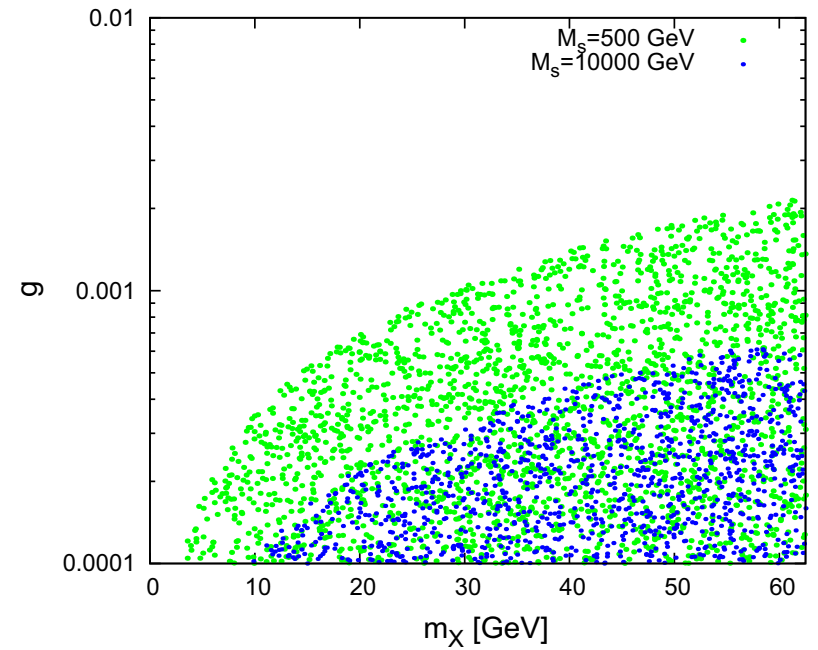

(b)

$g$ for different values of $M_{S}$, which are consistent with the experimental measurements of $\operatorname{Br}\left(H_{1} \rightarrow\right.$ Invisible $)$

and so the SM Higgs boson $H_{1}$ cannot decay to scalar DM. In Fig. 12a, we display $\operatorname{Br}\left(H_{1} \rightarrow\right.$ Invisible $)$ as a function of the fermionic DM mass for different values of the $g$ coupling. In this figure, we suppose $M_{\chi}<M_{H_{1}} / 2$ and assign other parameters such that $M_{H_{2}}<M_{H_{1}} / 2$. By using the ATLAS upper limit for invisible Higgs decay, we display the allowed range of parameter space in Fig. 12b in our model. Note that the main contribution to $\mathrm{Br}\left(\mathrm{H}_{1} \rightarrow\right.$ Invisible $)$ in the portion of parameter space which is consistent with the experimental limits arises from $\Gamma\left(H_{1} \rightarrow H_{2} H_{2}\right)$. This feature is shown in Fig. 13. This figure separately depicts the contribution of $\mathrm{Br}\left(\mathrm{H}_{1} \rightarrow\right.$ Invisible $)$ as a function of the fermionic DM mass for $\mathrm{Br}\left(\mathrm{H}_{1} \rightarrow \chi \chi\right), \mathrm{Br}\left(\mathrm{H}_{1} \rightarrow \mathrm{H}_{2} \mathrm{H}_{2}\right)$ and $\operatorname{Br}\left(H_{1} \rightarrow\right.$ total $)$. Comparing Fig. 13a, b implies for small values of $g$, which is consistent with experimental limits, that the main contributions of $\mathrm{Br}\left(\mathrm{H}_{1} \rightarrow\right.$ Invisible $)$ are coming from $\mathrm{Br}\left(\mathrm{H}_{1} \rightarrow \mathrm{H}_{2} \mathrm{H}_{2}\right)$. In our model, $\mathrm{M}_{H_{2}}$ generally depends on $g, M_{\chi}$ and $M_{s}$. Since $\Gamma\left(H_{1} \rightarrow H_{2} H_{2}\right)$ depends on $M_{H_{2}}$, in the allowed region of parameter space, we expect that the branching ratio of the invisible Higgs decay also depends on $M_{s}$. In Fig. 12b, we have shown for larger values of $M_{s}$ that the allowed area shrinks in the $g_{s}$ and $M_{\chi}$ plane.

In Fig. 14, the ranges of parameter space in the mass of fermionic DM and $g$ coupling, which are consistent with the observed relic density, are shown. Comparing Figs. 14 and $12 \mathrm{~b}$ shows that the allowed regions for invisible Higgs decay and the DM relic density do not overlap with each other. Since the highest contribution of the DM relic density arises from fermionic DM, for a small value of the $g$ coupling, the annihilation of DM to SM particles will be suppressed. This 


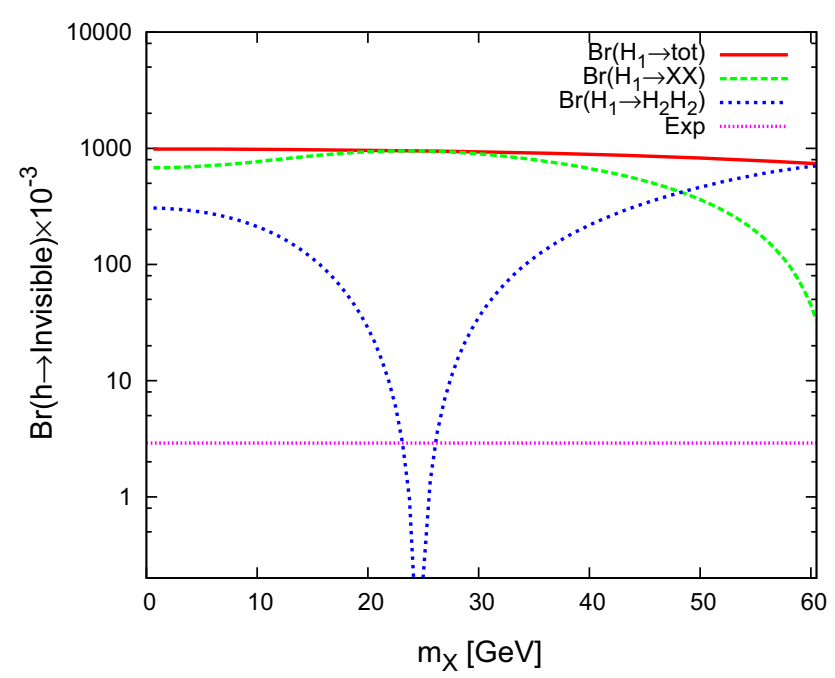

(a)

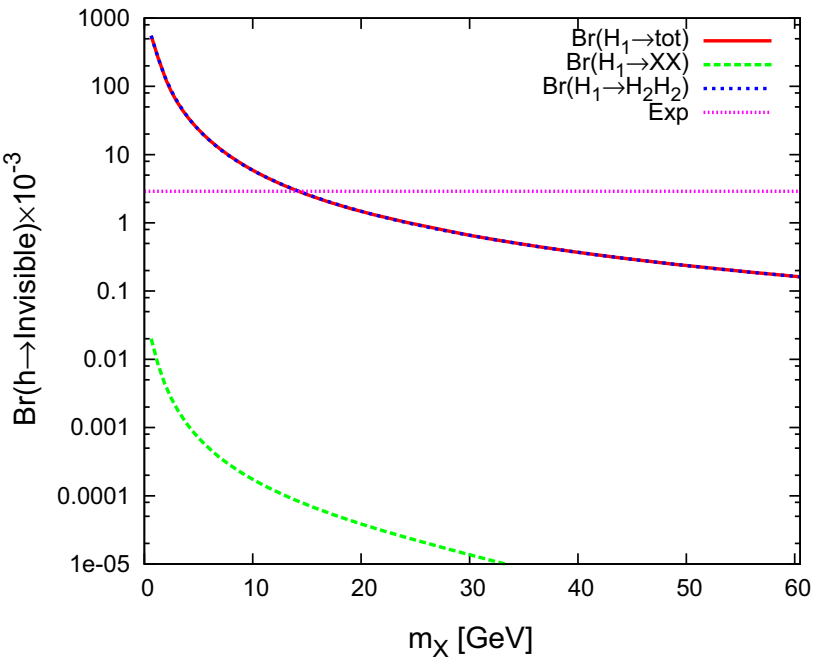

(b)

Fig. 13 a, b Different contributions of $\operatorname{Br}\left(H_{1} \rightarrow\right.$ Invisible) as a function of the fermionic DM mass for $g=0.1(\mathrm{~g}=0.0005)$ and $M_{s}=500 \mathrm{GeV}$

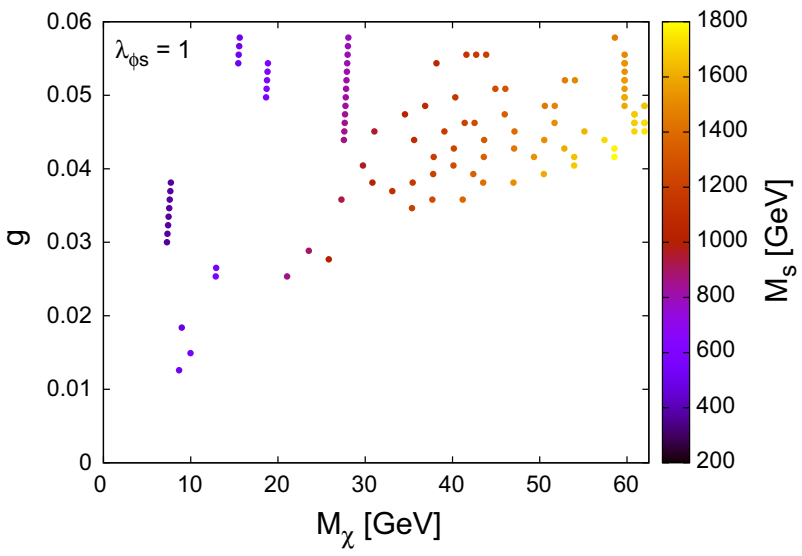

Fig. 14 Scatter points depict the ranges of parameter space in the mass of fermionic DM and $g$ coupling for different values of $M_{s}$, which are consistent with the observed relic density

means for a portion of the parameter space, which is consistent with invisible Higgs decay, the relic density exceeds the value of the Planck measurement. Therefore, in order to evade invisible Higgs constraints, one should assume that the fermionic DM mass is larger than $\frac{M_{H_{1}}}{2}$.

\section{Concluding remarks}

Motivated by the DM and hierarchy problems, we presented a scale-invariant extension of the SM. In order to have a scaleinvariant version of the SM with scalar DM, at least two more scalars must be added to the theory. Moreover, in the absence of additional fermionic fields, the model has a small number of independent parameters, which complicates the options of satisfying all theoretical and phenomenological constraints.
Given these conditions, we added a scalon field $\phi$, a scalar field $S$ and a fermionic field $\chi$ as two-component DM to SM. To summarize, the main novelty of this model, with respect to other two-component DM models, is the much smaller number of independent parameters due to the scale-invariant conditions.

In this analysis, the relic density of two-component DM was computed. We have shown that the highest part of the contribution of the DM relic density arises from fermionic DM. We have discussed the allowed regions in parameter space of our model consistent with the observed relic density.

We have also taken into account the constraints of indirect detection and direct detection of DM. In order to constrain the parameter space of our model, we also checked the limits from the self-interaction of DM. It is shown that the former analysis cannot put a constraint on the model in a large portion of parameter space. Only in the vicinity of the resonances in $M_{s} \simeq M_{H_{2}} / 2$ for scalar DM and $M_{\chi} \simeq M_{H_{2}} / 2$ or $M_{H_{1}} / 2$ for fermionic DM, the self-interaction scenario constrains the model.

Finally, we probed the limits from the invisible decay width of the Higgs. We have found that the viable regions in parameter space are in agreement with the upper limit on the invisible Higgs decay branching ratio. We compared the consistent region in parameter space for invisible Higgs decay with the relic density of the fermionic DM and show that in order to satisfy invisible Higgs constraints, the fermionic DM mass should be larger than $M_{H_{1}} / 2$.

Acknowledgements The authors would like to thank S. Paktinat for careful reading of the manuscript and the useful remarks.

Data Availability Statement This manuscript has no associated data or the data will not be deposited. [Authors' comment: Experimental 
Datasets derived from public resources and other data that support the findings of this study are available on request from the corresponding author.]

Open Access This article is distributed under the terms of the Creative Commons Attribution 4.0 International License (http://creativecomm ons.org/licenses/by/4.0/), which permits unrestricted use, distribution, and reproduction in any medium, provided you give appropriate credit to the original author(s) and the source, provide a link to the Creative Commons license, and indicate if changes were made.

Funded by SCOAP ${ }^{3}$.

\section{Appendix: DM self-interaction cross sections and decay rates}

In this appendix, we summarize the formulas of the selfinteracting cross sections for two components of DM and decay rates of two scalars Higgs.

The main contribution to $\sigma / M_{S}$ for scalar annihilation (processes $S S \longrightarrow S S[51]$ and $S S \longrightarrow \chi \chi$ ) in the nonrelativistic limit are given by

$$
\begin{aligned}
& \sigma(S S \rightarrow S S) / M_{s} \\
&=\frac{1}{64 \pi M_{s}^{3}} \mid \lambda_{s}+\frac{2 \lambda_{\phi s} M_{\chi} / g}{\sqrt{1+\left(\nu_{1} g / M_{\chi}\right)^{2}}} \frac{1}{s-M_{H_{2}}^{2}+i M_{H_{2}} \Gamma_{H_{2}}} \\
&-\left.\frac{2 \lambda_{\phi s} \nu_{1}}{\sqrt{1+\left(v_{1} g / M_{\chi}\right)^{2}}} \frac{1}{s-M_{H_{1}}^{2}+i M_{H_{1}} \Gamma_{H_{1}}}\right|^{2} \\
& \sigma(S S\rightarrow \chi \chi) / M_{s} \\
&= \frac{1}{32 \pi M_{s}}\left(1-\frac{4 M_{s}^{2}}{s}\right)^{-1 / 2}\left(1-\frac{4 M_{\chi}^{2}}{s}\right)^{3 / 2} \\
& \times \mid \frac{2 \lambda_{\phi s} M_{\chi}}{1+\left(v_{1} g / M_{\chi}\right)^{2}} \frac{1}{s-M_{H_{2}}^{2}+i M_{H_{2}} \Gamma_{H_{2}}} \\
&+\left.\frac{2 \lambda_{\phi s} v_{1}^{2} g^{2} / M_{\chi}}{1+\left(v_{1} g / M_{\chi}\right)^{2}} \frac{1}{s-M_{H_{1}}^{2}+i M_{H_{1}} \Gamma_{H_{1}}}\right|^{2}
\end{aligned}
$$

where $s$ is the usual Mandelstam variable and the decay rates for $\mathrm{H}_{2} \rightarrow \chi \chi$ and $\mathrm{H}_{2} \rightarrow S S$ are expressed by

$$
\begin{aligned}
& \Gamma\left(H_{2} \rightarrow \chi \chi\right)=\frac{g^{2} M_{H_{2}}}{2 \pi}\left(1-\frac{4 M_{\chi}^{2}}{M_{H_{2}}^{2}}\right)^{3 / 2}, \\
& \Gamma\left(H_{2} \rightarrow S S\right)=\frac{\lambda_{\phi s}^{2} M_{\chi}^{2}}{16 \pi g^{2} M_{H_{2}}}\left(1-\frac{4 M_{S}^{2}}{M_{H_{2}}^{2}}\right)^{1 / 2} .
\end{aligned}
$$

In the following, we calculate the self-interaction for the case of the Dirac fermionic DM which includes the processes $\chi \chi \longrightarrow \chi \chi$ and $\chi \chi \longrightarrow S S$. The cross sections of these processes are given by

$$
\begin{aligned}
& \sigma(\chi \chi\rightarrow \chi \chi) / M_{\chi} \\
&= \frac{g^{2} s}{16 \pi M_{\chi}}\left(1-\frac{4 M_{\chi}^{2}}{s}\right)^{2} \mid \frac{1}{\sqrt{1+\left(v_{1} g / M_{\chi}\right)^{2}}} \\
& \times \frac{1}{s-M_{H_{2}}^{2}+i M_{H_{2}} \Gamma_{H_{2}}} \\
&-\frac{g v_{1} / M_{\chi}}{\sqrt{1+\left(v_{1} g / M_{\chi}\right)^{2}}} \times\left.\frac{1}{s-M_{H_{1}}^{2}+i M_{H_{1}} \Gamma_{H_{1}}}\right|^{2}, \\
& \sigma(\chi \chi \frac{\lambda{ }_{\phi s}^{2} g^{2} M_{\chi}}{32 \pi}\left(1-\frac{4 M_{s}^{2}}{s}\right)^{1 / 2}\left(1-\frac{4 M_{\chi}^{2}}{s}\right)^{1 / 2} \\
& \quad \times \mid \frac{2 M_{\chi} / g}{1+\left(v_{1} g / M_{\chi}\right)^{2}} \frac{1}{s-M_{H_{2}}^{2}+i M_{H_{2}} \Gamma_{H_{2}}} \\
& \quad+\left.\frac{2 v_{1}^{2} g / M_{\chi}}{1+\left(v_{1} g / M_{\chi}\right)^{2}} \frac{1}{s-M_{H_{1}}^{2}+i M_{H_{1}} \Gamma_{H_{1}}}\right|^{2} .
\end{aligned}
$$

We also calculate the DM self-scattering cross sections for the processes $S \chi \longrightarrow S \chi$ in the non-relativistic limit. The cross sections can be written as

$$
\begin{aligned}
\sigma & (S \chi \rightarrow S \chi) / \bar{M} \simeq \frac{\lambda_{\phi s}^{2} M_{\chi}^{4}}{8 \pi p^{2}\left(M_{\chi}+M_{s}\right)^{3}} \\
& \times\left[\left(\frac{1}{\left(m_{H_{2}}^{2}\right)}-\frac{1}{\left(4 p^{2}+m_{H_{2}}^{2}\right)}\right)\left(\frac{2 M_{\chi} / g}{1+\left(v_{1} g / M_{\chi}\right)^{2}}\right)^{2}\right. \\
& \left.+\left(\frac{1}{\left(m_{H_{1}}^{2}\right)}-\frac{1}{\left(4 p^{2}+m_{H_{1}}^{2}\right)}\right)\left(\frac{2 v_{1}^{2} g / M_{\chi}}{1+\left(v_{1} g / M_{\chi}\right)^{2}}\right)^{2}\right] \\
& \times\left[\left(\frac{E_{p}^{2}+m_{\chi}^{2}}{\sqrt{E_{p}^{2}-m_{\chi}^{2}}} \sqrt{E_{k}^{2}-m_{\chi}^{2}}\right]\right.
\end{aligned}
$$

where $p, E_{p}$ and $E_{k}$ are the momentum of the initial fermionic DM, the energy of the initial fermionic DM and the energy of the final fermionic DM, respectively.

We also calculate the following formulas for the decay rates of $H_{1} \rightarrow \chi \chi, H_{1} \rightarrow S S$ and $H_{1} \rightarrow H_{2} H_{2}$ :

$$
\begin{aligned}
& \Gamma\left(H_{1} \rightarrow \chi \chi\right)=\frac{M_{H_{1}} a_{H_{1} \chi \chi}^{2}}{2 \pi}\left(1-\frac{4 M_{\chi}^{2}}{M_{H_{1}}^{2}}\right)^{3 / 2}, \\
& \Gamma\left(H_{1} \rightarrow S S\right)=\frac{a_{H 1 S S}^{2}}{16 \pi M_{H_{1}}}\left(1-\frac{4 M_{s}^{2}}{M_{H_{1}}^{2}}\right)^{1 / 2}, \\
& \Gamma\left(H_{1} \rightarrow H_{2} H_{2}\right)=\frac{a_{H_{1} H_{2} H_{2}}^{2}}{16 \pi M_{H_{1}}}\left(1-\frac{4 M_{H_{2}}^{2}}{M_{H_{1}}^{2}}\right)^{1 / 2},
\end{aligned}
$$


where

$$
\begin{aligned}
a_{H_{1} \chi \chi} & =\frac{g^{2} v_{1}}{\sqrt{\left(g^{2} v_{1}^{2}+M_{\chi}^{2}\right)}}, \\
a_{H 1 S S} & =\frac{2 v_{1} \lambda_{\phi s}}{\left(1+\left(v_{1} g / M_{\chi}\right)^{2}\right)^{1 / 2}}+\frac{\left(M_{s}^{2}-2 \lambda_{\phi s} M_{\chi}^{2} / g^{2}\right)}{v_{1} \sqrt{1+\left(v_{1} g / M_{\chi}\right)^{2}}}, \\
a_{H 1 H_{2} H_{2}} & =\frac{M_{H_{1}}^{2}}{2\left(1+\left(v_{1} g / M_{\chi}\right)^{2}\right)^{5 / 2}}\left[v_{1}^{4}\left(\frac{g}{M_{\chi}}\right)^{5}-\frac{g}{M_{\chi}}\right] .
\end{aligned}
$$

\section{References}

1. A.M. Sirunyan et al., [CMS Collaboration]. JHEP 1712, 142 (2017). https://doi.org/10.1007/JHEP12(2017)142. [arXiv:1707.06193 [hep-ex]]

2. C. Autermann, [ATLAS and CMS Collaborations]. EPJ Web Conf. 164, 01028 (2017). https://doi.org/10.1051/epjconf/ 201716401028

3. CMS Collaboration [CMS Collaboration], CMS-PAS-SUS-17-012

4. M. Aaboud et al., [ATLAS Collaboration]. Phys. Rev. D 98(3), 032009 (2018). https://doi.org/10.1103/PhysRevD.98. 032009. [arXiv:1804.03602 [hep-ex]]

5. M. Aaboud et al., [ATLAS Collaboration]. Phys. Rev. D 97(9), 092006 (2018). https://doi.org/10.1103/PhysRevD.97. 092006. [arXiv: 1802.03158 [hep-ex]]

6. M. Aaboud et al., [ATLAS Collaboration]. JHEP 1806, 107 (2018). https://doi.org/10.1007/JHEP06(2018)107. [arXiv:1711.01901 [hep-ex]]

7. W. A. Bardeen, FERMILAB-CONF-95-391-T

8. E. Gildener, S. Weinberg, Phys. Rev. D 13, 3333 (1976). https:// doi.org/10.1103/PhysRevD.13.3333

9. S. R. Coleman, E.J., Phys. Rev. D 7, 1888 (1973)

10. C. Englert, J. Jaeckel, V.V. Khoze, M. Spannowsky, JHEP 1304, 060 (2013). https://doi.org/10.1007/JHEP04(2013)060. [arXiv:1301.4224 [hep-ph]]

11. M. Heikinheimo, A. Racioppi, M. Raidal, C. Spethmann, K. Tuominen, Mod. Phys. Lett. A 29, 1450077 (2014). https://doi. org/10.1142/S0217732314500771. [arXiv:1304.7006 [hep-ph]]

12. K.A. Meissner, H. Nicolai, Phys. Lett. B 648, 312 (2007). [arXiv: hep-th/0612165]

13. R. Foot, A. Kobakhidze, K.L. McDonald, R.R. Volkas, Phys. Rev. D 77, 035006 (2008). [arXiv:0709.2750]

14. C. Cosme, J.G. Rosa, O. Bertolami, JHEP 1805, 129 (2018). https:// doi.org/10.1007/JHEP05(2018)129. [arXiv:1802.09434 [hep-ph]]

15. K. Kannike, M. Raidal, C. Spethmann, H. Veerme, JHEP 1704, 026 (2017). https://doi.org/10.1007/JHEP04(2017)026. [arXiv:1610.06571 [hep-ph]]

16. A. Karam, K. Tamvakis, Phys. Rev. D 94(5), 055004 (2016). https:// doi.org/10.1103/PhysRevD.94.055004. [arXiv:1607.01001 [hep$\mathrm{ph}]]$

17. V.V. Khoze, A.D. Plascencia, JHEP 1611, 025 (2016). https://doi. org/10.1007/JHEP11(2016)025. arXiv:1605.06834 [hep-ph]

18. A. Ahriche, A. Manning, K.L. McDonald, S. Nasri, Phys. Rev. D 94(5), 053005 (2016). https://doi.org/10.1103/PhysRevD.94. 053005. arXiv:1604.05995 [hep-ph]

19. A. Karam, K. Tamvakis, Phys. Rev. D 92(7), 075010 (2015). https://doi.org/10.1103/PhysRevD.92.075010. arXiv:1508.03031 [hep-ph]

20. S. Benic, B. Radovcic, JHEP 1501, 143 (2015). https://doi.org/10. 1007/JHEP01(2015)143. arXiv:1409.5776 [hep-ph]
21. J.H. Huh, J.E. Kim, B. Kyae, Phys. Rev. D 79, 063529 (2009). https://doi.org/10.1103/PhysRevD.79.063529. arXiv:0809.2601 [hep-ph]

22. S. Bhattacharya, A. Drozd, B. Grzadkowski, J. Wudka, JHEP 1310, 158 (2013). https://doi.org/10.1007/JHEP10(2013)158. arXiv:1309.2986 [hep-ph]

23. A. Biswas, D. Majumdar, P. Roy, JHEP 1504, 065 (2015). https:// doi.org/10.1007/JHEP04(2015)065. arXiv:1501.02666 [hep-ph]

24. A. Biswas, D. Majumdar, A. Sil, P. Bhattacharjee, JCAP 1312, 049 (2013). https://doi.org/10.1088/1475-7516/2013/12/ 049. arXiv:1301.3668 [hep-ph]

25. S. Esch, M. Klasen, C.E. Yaguna, JHEP 1409, 108 (2014). https:// doi.org/10.1007/JHEP09(2014)108. arXiv:1406.0617 [hep-ph]

26. A. Biswas, J. Phys. G 43(5), 055201 (2016). https://doi.org/10. 1088/0954-3899/43/5/055201. arXiv:1412.1663 [hep-ph]

27. L. Bian, R. Ding, B. Zhu, Phys. Lett. B 728, 105 (2014). https://doi. org/10.1016/j.physletb.2013.11.034. arXiv:1308.3851 [hep-ph]

28. Y. Daikoku, H. Okada, T. Toma, Prog. Theor. Phys. 126, 855 (2011). https://doi.org/10.1143/PTP.126.855. arXiv:1106.4717 [hep-ph]

29. L. Bian, T. Li, J. Shu, X.C. Wang, JHEP 1503, 126 (2015). https:// doi.org/10.1007/JHEP03(2015)126. arXiv:1412.5443 [hep-ph]

30. A. Dutta Banik, M. Pandey, D. Majumdar, A. Biswas, Eur. Phys. J. C 77(10), 657 (2017). https://doi.org/10.1140/epjc/ s10052-017-5221-y. arXiv:1612.08621 [hep-ph]

31. K. Belotsky, M. Khlopov, C. Kouvaris, M. Laletin, Int. J. Mod. Phys. D 24(13), 1545004 (2015). https://doi.org/10.1142/ S0218271815450042. arXiv:1508.02881 [astro-ph.HE]

32. M. Aoki, T. Toma, JCAP 1701(01), 042 (2017). https://doi.org/10. 1088/1475-7516/2017/01/042. arXiv:1611.06746 [hep-ph]

33. M. Pandey, D. Majumdar, K.P. Modak, JCAP 1806(06), 023 (2018). https://doi.org/10.1088/1475-7516/2018/06/023. arXiv:1709.05955 [hep-ph]

34. S. Peyman Zakeri, S. Mohammad Moosavi Nejad, M. Zakeri, S. Yaser Ayazi, Chin. Phys. C 42(7), 073101 (2018). https://doi.org/ 10.1088/1674-1137/42/7/073101. arXiv:1801.09115 [hep-ph]

35. K. Ghorbani, H. Ghorbani, JHEP 1604, 024 (2016). https://doi.org/ 10.1007/JHEP04(2016)024. arXiv:1511.08432 [hep-ph]

36. G. Belanger, F. Boudjema, A. Pukhov, A. Semenov, Comput. Phys. Commun. 192, 322 (2015). https://doi.org/10.1016/j.cpc.2015.03. 003. arXiv:1407.6129 [hep-ph]

37. A. Semenov, Comput. Phys. Commun. 180, 431 (2009). https:// doi.org/10.1016/j.cpc.2008.10.012. arXiv:0805.0555 [hep-ph]

38. P.A.R. Ade et al., (Planck Collaboration). Astron. Astrophys 571, A31 (2014)

39. E. Aprile et al., [XENON Collaboration]. Phys. Rev. Lett. 121(11), 111302 (2018). https://doi.org/10.1103/PhysRevLett.121.111302. arXiv:1805.12562 [astro-ph.CO]

40. D.S. Akerib et al., [LUX Collaboration]. Phys. Rev. Lett. 118(2), 021303 (2017). https://doi.org/10.1103/PhysRevLett.118.021303. arXiv: 1608.07648 [astro-ph.CO]

41. X.G. He, T. Li, X.Q. Li, J. Tandean, H.C. Tsai, Phys. Rev. D 79, 023521 (2009). https://doi.org/10.1103/PhysRevD.79.023521. arXiv:0811.0658 [hep-ph]

42. E. Aprile et al., [XENON Collaboration]. JCAP 1604(04), 027 (2016). https://doi.org/10.1088/1475-7516/2016/04/027. arXiv:1512.07501 [physics.ins-det]

43. M. Ackermann et al., [Fermi-LAT Collaboration]. Phys. Rev. Lett. 115(23), 231301 (2015). https://doi.org/10.1103/PhysRevLett. 115.231301. arXiv:1503.02641 [astro-ph.HE]

44. O. Adriani et al., [PAMELA Collaboration]. Phys. Rev. Lett. 111, 081102 (2013). https://doi.org/10.1103/PhysRevLett.111.081102. arXiv:1308.0133 [astro-ph.HE]

45. M. Aguilar et al., [AMS Collaboration]. Phys. Rev. Lett. 117(9), 091103 (2016). https://doi.org/10.1103/PhysRevLett.117.091103 
46. M.G. Aartsen et al., [IceCube Collaboration]. Eur. Phys. J. C 77(3), 146 (2017). https://doi.org/10.1140/epjc/s10052-017-4689-9. arXiv:1612.05949 [astro-ph.HE]

47. M.G. Aartsen et al., [IceCube Collaboration]. Eur. Phys. J. C 77(2), 82 (2017). https://doi.org/10.1140/epjc/s10052-016-4582-y. arXiv:1609.01492 [astro-ph.HE]

48. G. Steigman, B. Dasgupta, J.F. Beacom, Phys. Rev. D 86, 023506 (2012). https://doi.org/10.1103/PhysRevD.86.023506. arXiv:1204.3622 [hep-ph]

49. F. Kahlhoefer, K. Schmidt-Hoberg, J. Kummer, S. Sarkar, Mon. Not. R. Astron. Soc. 452(1), L54 (2015). https://doi.org/10.1093/ mnrasl/slv088. arXiv:1504.06576 [astro-ph.CO]

50. M. Kaplinghat, S. Tulin, H.B. Yu, Phys. Rev. Lett. 116(4), 041302 (2016). https://doi.org/10.1103/PhysRevLett.116.041302. arXiv:1508.03339 [astro-ph.CO]
51. R. Campbell, S. Godfrey, H.E. Logan, A.D. Peterson, A. Poulin, Phys. Rev. D 92(5), 055031 (2015)

52. S. Dittmaier et al., [LHC Higgs Cross Section Working Group], https://doi.org/10.5170/CERN-2011-002. arXiv:1101.0593 [hep$\mathrm{ph}]$

53. CMS Collaboration [CMS Collaboration], CMS-PAS-HIG-14-038

54. The ATLAS collaboration [ATLAS Collaboration], ATLASCONF-2015-004

55. G. Belanger, B. Dumont, U. Ellwanger, J.F. Gunion, S. Kraml, Phys. Lett. B 723, 340 (2013). https://doi.org/10.1016/j.physletb. 2013.05.024. arXiv:1302.5694 [hep-ph]

56. S. Heinemeyer et al., [LHC Higgs Cross Section Working Group], https://doi.org/10.5170/CERN-2013-004. arXiv:1307.1347 [hep$\mathrm{ph}]$ 